\title{
Co-allocation to overlapping dendritic branches in the retrosplenial cortex integrates memories across time
}

Megha Sehgal ${ }^{1}$, Daniel Almeida Filho ${ }^{1}$, George Kastellakis², Sungsoo Kim³ ${ }^{3}$, Jinsu Lee ${ }^{2}$, Sunaina Martin ${ }^{1}$, Irene Davila Mejia ${ }^{1}$, Asli Pekcan ${ }^{1}$, Shan Huang ${ }^{1}$, Ayal Lavi ${ }^{1}$, Won Do Heo ${ }^{3}$, Panayiota Poirazi $^{2}$, Joshua T. Trachtenberg ${ }^{1}$, Alcino J. Silva ${ }^{1}$

${ }^{1}$ Departments of Neurobiology, Psychiatry and Psychology \& Integrative Center for Learning and Memory, UCLA, Los Angeles, CA.

${ }^{2}$ Institute of Molecular Biology and Biotechnology (IMBB), Foundation for Research and Technology, Hellas (FORTH), Vassilika Vouton, PO Box 1385, GR 70013 Heraklion, Crete, Greece.

${ }^{3}$ Department of Biological Sciences, Korea Advanced Institute of Science and Technology, Daejeon, Republic of Korea.

*Corresponding author. Email: silvaa@mednet.ucla.edu 


\begin{abstract}
Events occurring close in time are often linked in memory, providing an episodic timeline and a framework for those memories. Recent studies suggest that memories acquired close in time are encoded by overlapping neuronal ensembles, but whether dendritic plasticity plays a role in linking memories is unknown. Using activity-dependent labeling and manipulation, as well as longitudinal one- and two-photon imaging of RSC somatic and dendritic compartments, we show that memory linking is not only dependent on ensemble overlap in the retrosplenial cortex, but also on branchspecific dendritic allocation mechanisms. These results demonstrate a causal role for dendritic mechanisms in memory integration and reveal a novel set of rules that govern how linked, and independent memories are allocated to dendritic compartments.
\end{abstract}

One-Sentence Summary: Dendritic allocation mechanisms link distinct memories across time 
Memory formation is a dynamic process, where single memories are stored, updated, and integrated within the framework of other pre-existing memories to drive adaptive behavior (1-3). Recent studies have revealed that the overlap between the neuronal ensembles encoding different memories can link them, such that the recall of one leads to the recall of the other (4-6). However, memory formation and retrieval are mediated by dendritic processes $(7,8)$. Yet, our understanding of the dendritic mechanisms that regulate the encoding of linked memories is limited. Experience-dependent dendritic plasticity is branch-specific (9-12) and potentiation of dendritic spines for a limited period affects future plasticity at nearby spines on the same dendritic branch $(10,13)$. Therefore, we hypothesized that two memories acquired close in time would be allocated to an overlapping population of dendritic branches, and that this mechanism drives memory linking (14). To test this hypothesis, we investigated the role of the retrosplenial cortex (RSC) dendritic allocation mechanisms in contextual memory linking. We chose the RSC because of its important role in spatial and contextual memory processing $(15,16)$, as well as ease of access for longitudinal, non-invasive imaging of neuronal ensembles and dendritic segments.

The overlap between dorsal CA1 neuronal ensembles encoding two contextual memories (neuronal co-allocation) is critical for linking these memories (4). However, it is unclear if this is limited to certain key brain regions, such as the CA1, or extends to the other areas within the neural circuit involved in contextual memory formation. Thus, we first investigated whether RSC neuronal ensembles encoding memories of two contexts close in time (i.e., linked memories) also display a higher overlap than two ensembles encoding memories further apart (i.e., independent memories). We used head-mounted miniature microscopes to image GCaMP6f-mediated calcium dynamics in RSC neurons (figure 1a-d, 4599 putative RSC neurons, $132.9 \pm 11.6$ neurons per session) while mice explored different contexts. We found a greater overlap between the RSC neuronal ensembles encoding two contexts explored on the same day ( 5 hours apart), than between the neuronal ensembles encoding two contexts explored one week apart (figure 1e). Notably, the increased overlap between neuronal ensembles for memories acquired 5 hours 
versus one week apart was not due to differences in the size of these ensembles (figure S1). These data indicate that similar to the results in dorsal CA1 (4), RSC neurons also encode temporally proximate contextual memories using overlapping neuronal populations. These results provide strong evidence that the co-allocation of temporally proximate memories to overlapping neuronal ensembles is a universal mechanism for memory linking.

We reasoned that if, similar to CA1 (4), transient increases in intrinsic excitability in RSC drive neuronal ensemble overlap of linked memories, then the firing rate of RSC neurons should be similar for contexts explored close in time. We found that RSC neurons maintained a similar firing rate for contexts explored within 5 hours compared to contexts explored 7 days apart (figure If and S2). Since RSC neurons encode distinct contexts using a firing rate code (16), it is likely that neuronal firing dynamics within the RSC also impact the ability to decode context identity. Indeed, a Naïve Bayes (NB) classifier performed better at distinguishing sessions recorded a week apart relative to those recorded within the same day (figure $1 \mathrm{~g}$ and S3). Finally, we investigated the coactivity patterns of RSC neurons during these context explorations. Theoretical as well as experimental models suggest that groups of neurons with synchronized activity encode task-relevant information in the hippocampus, cortical, and subcortical regions (17-24). However, the significance of such coactivity patterns during memory formation within the RSC is unclear. Therefore, we calculated the pairwise correlation (PWC) for each pair of RSC neurons within each session (PWC map) and found that these correlations are generally stable across imaging sessions (figure S4). However, the across-session stability of these PWC maps is higher when contexts are explored on the same day compared to one week apart (figure $1 \mathrm{~h}$ ), indicating that RSC neurons maintain patterns of coactivity when contexts are explored within the same day. Such synchronous firing may be key for the linking of contextual memories within the RSC. Together, these data indicate that overlapping RSC ensembles are activated when contextual memories are acquired close in time, and that the dynamic activity of these overlapping ensembles may play a critical role in linking different contextual memories. 
To investigate the causal role of RSC neuronal co-allocation in linking contextual memories, we used the TetTag system (25) to tag and manipulate the RSC neuronal ensembles activated during context exposures (figure 2a). We confirmed that optogenetic reactivation of the RSC ensemble underlying a single contextual fear memory induces fear expression in an otherwise neutral and novel context (figure S5 and 15). When two contextual memories are acquired close in time, and one is paired with a fearful stimulus, the mice also consider the second neutral but 'linked' context as fearful (i.e., the two memories are linked) (4). We asked whether reactivation of RSC neurons engaged during exploration of the 'linked' context after contextual memory linking was sufficient to elicit freezing in mice (figure 2b). Indeed, optogenetic reactivation of the 'linked' context's neuronal ensemble in RSC alone was sufficient to trigger freezing in mice exploring a novel context (figure 2b). The differences in optogenetic reactivation of fear between the two groups (i.e., TetTag mice and controls) did not stem from differences in contextual learning or linking of contextual memories. We tested the same mice in three different contexts on three consecutive days: the 'linked context', another novel context, and the 'shock context' to assess their levels of memory linking, memory generalization, and conditioned responses, respectively. Both groups of mice displayed high levels of freezing in 'linked' (Context A) as well as 'shock' (Context B) contexts in comparison to freezing in the novel context (figure S6). Thus, both groups of mice learned the context-shock association and linked the fearful context to the previously explored context ('linked' context), but only mice where the 'linked' context ensemble was reactivated using the TetTag system displayed fear in a novel context. First, these data indicate that reactivating the memory of a 'linked', but otherwise neutral context, was sufficient to elicit a conditioned response, a result that supports our hypothesis that recall of one linked memory results in the recall of the other. Second, these findings also demonstrate that manipulation of neuronal ensembles just within the RSC can drive contextual memory linking.

While two contexts explored within a day are linked, contexts explored 2 or 7 days apart are not allocated to overlapping neuronal ensembles, and therefore are not linked (4). We asked 
if we could link two distant contextual memories (acquired 2 days apart) by artificially biasing the involvement of a specific RSC neuronal ensemble in the encoding of both memories. With the TetTag system (25), we tagged the RSC neuronal ensemble activated during a context exploration (Context $A$ ) and reactivated this ensemble the next day, one day before exposure to another context (Context $\mathrm{B}$, figure $2 \mathrm{c}$ ). We reasoned that this would optogenetically reactivate the first memory, maintain the increase in neuronal excitability, and therefore force the recruitment of this same ensemble (26) during the exploration of another context a day later. While two contexts explored 2 days apart are normally not linked, this optogenetic reactivation of the first contextual memory was sufficient to bridge this 2-day gap and drive linking of two otherwise independent contextual memories (figure 2c). We further confirmed the role of neuronal ensemble overlap in the RSC using a chemogenetic (Lentivirus DREADD (4)) system. We biased neuronal coallocation of two distinct contextual memories by enhancing the neuronal excitability in a sparse population of RSC neurons before each context exploration (2 days apart, figure S7). Like the optogenetic manipulation, artificially biasing co-allocation of two distant contextual memories to overlapping RSC ensembles using chemogenetics drives the linking of the two memories, such that the mice showed comparable freezing in both contexts (Context A and B). Together, these data demonstrate that neuronal ensemble overlap in RSC is sufficient to link the memories of two distinct contexts.

Ours and previous results (4) demonstrate that the allocation of contextual memories to overlapping neuronal ensembles is critical for linking those memories. Within these overlapping ensembles, linked memories are thought to be encoded by distinct synaptic changes that allow the memories to maintain their distinct identities (27). There are at least three dendritic hypotheses that could account for contextual memory linking. First, linked memories may be allocated to different dendritic branches within the encoding neurons (dis-allocation). Second, it is possible that linked memories are randomly allocated to the dendritic branches within the encoding neurons. Alternatively, since experience-dependent dendritic plasticity is highly 
localized and can affect future plasticity at nearby spines on the same dendritic segment under certain conditions $(10,13)$, it is also conceivable that, following the first context exposure, branchspecific changes in dendritic plasticity temporarily bias the activation of the same dendritic segments during a subsequent context exposure (14). In this latter scenario, distinct synaptic changes on the same dendritic branches could drive the co-activation, and therefore the linking of the two memories. To distinguish between these different hypotheses, we used two-photon microscopy to perform longitudinal calcium imaging of the same RSC apical dendrites (figure 3ab) while mice explored distinct contexts in a head-fixed setting (figure S8). Consistent with our results with RSC neuronal activation, we found that the same dendritic segments were preferentially reactivated as mice explored two distinct contexts within the same day, but not a week apart (figure 3c-e). These data are consistent with the hypothesis that local dendritic mechanisms govern the allocation of two contextual memories encoded close in time to the same dendritic segments (14). Although backpropagating action potentials (bAPs) are not always evident at RSC apical dendritic branches (28), we acknowledge that our results could be confounded by bAPs, and therefore used structural imaging of RSC dendrites to confirm and extend these findings.

Given that overlapping dendritic segments are activated when encoding contexts that are experienced close in time, we next investigated whether learning-related spine dynamics were also evident on the same dendritic segments when contextual memories are acquired close in time. Within the RSC, contextual memory formation is accompanied by structural plasticity at dendritic spines such that behavioral performance is positively correlated with clustered spine addition on small stretches $(\sim 5 \mu \mathrm{m})$ of a dendritic segment (29). We used in vivo two-photon microscopy to image spines on the RSC apical dendrites of Thy1-YFP-H mice following multiple context exposures (figure $4 a-c$ ). As structural imaging involved mice to be imaged under anesthesia, we confirmed that mice were still able to link distinct contexts when context exposures are followed by prolonged periods of anesthesia ( 40mins, figure S9). We quantified the spines 
added or lost following each context exposure and found that, relative to spine dynamics during a baseline period, novel context exposure does not change overall spine addition, spine loss, or spine turnover (figure S10a, b). However, following context exposure, new spines were added in clusters (i.e., within $5 \mu \mathrm{m}$ of each other); and a shuffling analysis showed that the number of these clustered spines was significantly above chance levels ( $p=0.009$; figure S10c). In contrast, clustered spine addition in the control group - that went through all the same imaging procedures, but that were never exposed to a novel context - was at chance levels ( $p=0.168$; figure S10c). Hence, consistent with our previous findings (29), novel context exploration results in clustered plasticity in RSC dendrites.

Since our results showed reactivation of the same dendrites during context exposures close in time, we next investigated the possibility that spines added following these context exposures also tend to be added to the same dendritic segments (figure $4 \mathrm{~d}$ ). We found a positive correlation between the number of spines added to the same dendritic segments following two context exposures experienced 5 hours apart (figure 4d). In contrast, the number of spines added to the same dendritic segments following context exposures one week apart were not correlated (figure 4d). Spine addition was also not correlated in the control group that experienced the same imaging procedures, but no contextual exposures, at either the 5 hours or 7 day time intervals (figure 4e). Furthermore, the number of spines lost was not correlated whether the imaging sessions were conducted 7 days or 5 hours apart in both the control and experimental groups (figure S11). These data indicate that spine addition is biased to the same RSC dendritic segments when contextual memories are linked (acquired 5 hours apart), but not when these memories are independent (acquired 7 days apart).

Finally, we also analyzed spine dynamics looking for co-allocation of clustered spines (i.e., whether dendritic segments that gained clustered spines during a context exposure were also the ones that gained clustered spines during a previous context exposure). We found that the probability of the same dendritic segments gaining spine clusters following exposures to two 
contexts was at chance levels when these contexts were explored 7 days apart $(p=0.4)$. Importantly, spine clusters were more likely to be added to the same dendritic segments when contexts were explored within the same day (5 hours apart; $p=0.03$, figure $4 \mathrm{f}$ ). In contrast, the addition of clustered spines in the control group was random: the probability of the same dendritic segments gaining spine clusters during two imaging sessions was at chance levels whether the imaging sessions were 5 hours or 7 days apart ( $p=0.28$ and 0.5 respectively; figure $4 \mathrm{~g}$ ). Together, the structural, as well as functional imaging data from RSC dendrites, indicate that the same dendrites are recruited to encode contextual memories formed close in time (i.e., linked memories).

Next, we tested whether such dendritic co-allocation is sufficient for linking contextual memories. To investigate the behavioral significance of RSC dendritic co-allocation, we developed an optogenetic tool for tagging and manipulating previously activated dendritic segments: We leveraged the activity-dependent labeling of the cFos-tTA system, and combined it with the dendritic targeting element (DTE) of Arc mRNA, which is selectively targeted and locally translated in activated dendritic segments following learning (figure 5a,b)(30, 31). This new approach allowed us to manipulate dendritic activity by expressing a light-sensitive ion channel (channelrhodopsin) in recently activated dendritic segments of RSC neurons tagged during a context exposure. We first assessed whether activation of dendritic segments tagged in this manner results in somatic activation. RSC dendrites activated during contextual memory formation were tagged and reactivated in a novel context (figure S5g-h). Unlike our somatic manipulations, tagging and activation of dendritic compartments alone was not sufficient to elicit fear expression in a novel context indicating that channelrhodopsin-mediated dendritic activation is insufficient to depolarize the soma and therefore fails to elicit a behavioral response. Hence, combining the cFos-tTA system with DTE allowed us to study the role of previously active dendrites (in the absence of somatic activation) in memory linking. 
Next, we asked whether artificially biasing dendritic allocation, similar to our neuronal manipulations (figure $2 \mathrm{c}$ and $\mathrm{S7}$ ), is sufficient to link two contextual memories, which would otherwise be independent. We tagged active dendrites during the first context exploration (Context $A$ ) and reactivated these dendrites the next day while the mice were in their home cage. One day after this reactivation (i.e., two days after the exploration of Context A), we exposed the mice to another novel context (Context B). As we have shown above, two contexts explored 2 days apart are normally not linked (figure 2c and S7) (4). However, similar to the reactivation of Context A neurons (figure 2c), the reactivation of dendrites first activated in Context A is sufficient to link Context A and Context B (the shock context): freezing in the 'linked context' (Context A) was higher than freezing in a 'novel' context and similar to freezing in the shock context (Context B). Hence, reactivation of dendrites tagged during exploration of one context is sufficient to link that context to another independent context (figure 5c). These results demonstrate a causal role for RSC dendritic mechanisms in the allocation as well as linking of contextual memories and reveal a novel set of rules that govern how linked, and independent memories are allocated to various dendritic compartments.

Our data thus far suggests that synergism between somatic and dendritic mechanisms sculpts memory allocation within the RSC to regulate linking of memories. To understand whether linking of memories that are acquired close in time is even possible in the absence of dendritic mechanisms, we adapted a network model $(14,29)$ to incorporate somatic as well as dendritic allocation mechanisms (see methods, figure S12a, b). As with our experimental data, the network model shows that neuronal (figure S12c) as well as dendritic overlap (figure S12d,e) is higher when two memories are acquired close in time ( 5 hours vs 2 or 7 days apart). When linked memories are recalled (i.e., memories acquired 5 hours apart), they maintain higher neuronal overlap indicating co-recall and linking of these memories (figure S12f). Finally, we asked how these neuronal and dendritic overlap measures change in the absence of dendritic allocation and plasticity mechanisms. Remarkably, neuronal as well as dendritic overlap during encoding is 
reduced when the model lacks dendritic mechanisms (figure S12c-e). More importantly, the lack of dendritic mechanisms in the model abolished co-recall or linking of memories (neuronal overlap during recall is the same whether memories are acquired 5 hours, 2 days or 7 days apart). Together, our data indicate that dendritic allocation mechanisms are necessary (figure S12) and sufficient (figure 5) for linking memories acquired close in time.

Our findings demonstrate that RSC neuronal and dendritic mechanisms play a causal role in linking contextual memories. Since RSC is one of the first brain regions to demonstrate Alzheimer's-related dysfunction $(32,33)$, and memory linking is affected early during aging (4), our findings have clinical implications for understanding age-related cognitive decline and associated pathological conditions. Furthermore, we demonstrate that in addition to neuronal ensemble overlap, local dendritic rules further sculpt the allocation of memories to dendritic segments, such that temporally proximate (i.e., linked) memories are likely to be allocated to the same dendritic segments, while temporally distant (i.e., independent) memories are not. We leverage activity-dependent targeting of dendritic segments to demonstrate that biasing memory allocation to the same dendritic segments is sufficient to link these memories and computational modeling to demonstrate that these dendritic mechanisms are necessary for memory linking. Altogether, the findings presented here demonstrate that branch-specific dendritic mechanisms are critical for linking memories. Within the dendritic arbor, inputs from distinct pathways are organized on distinct dendritic domains (34), and this organization along with our current findings suggest that only inputs impinging on the same dendritic compartments might be linked while other inputs remain independent. It is also likely that aberrant dendritic and synaptic plasticity mechanisms prevent memory integration in pathological states $(35,36)$. 
bioRxiv preprint doi: https://doi org/10.1101/2021.10.28.466343; this version posted December 7, 2021. The copyright holder for this preprint (which was not certified by peer review) is the author/funder, who has granted bioRxiv a license to display the preprint in perpetuity. It is made available under aCC-BY-ND 4.0 International license.

A

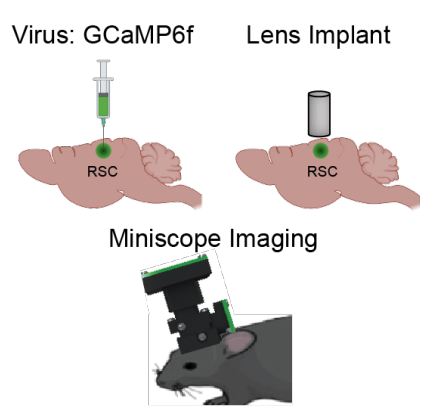

C

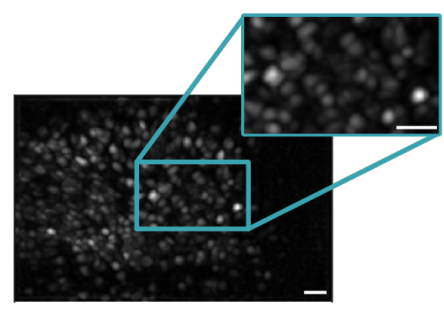

B

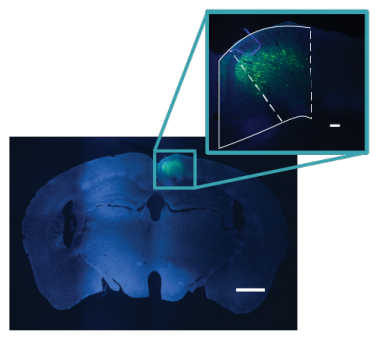

D

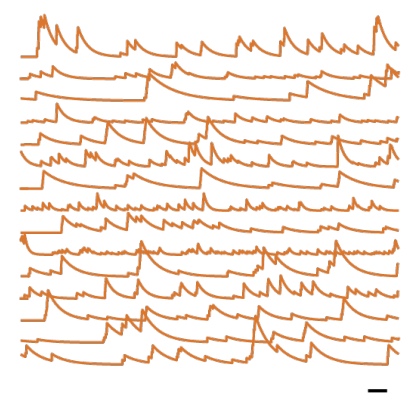

$\mathbf{E}$

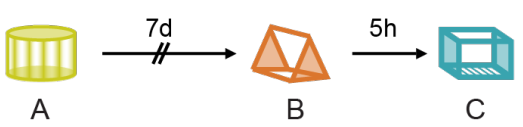

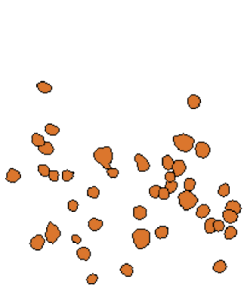

$7 d$

F

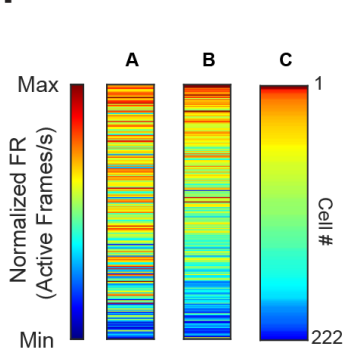

H

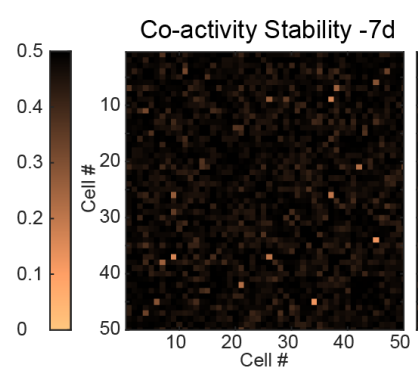

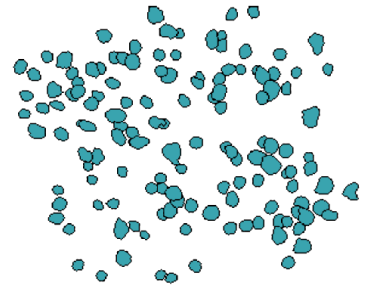

$5 h$

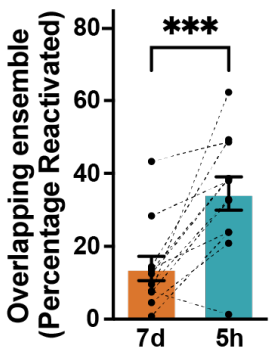

G
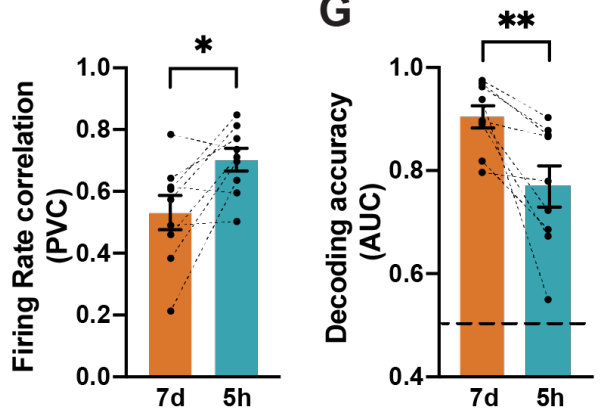

I
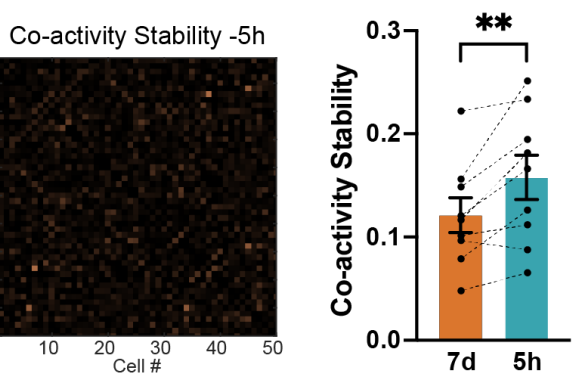


\section{Figure 1. Overlapping RSC ensembles are recruited to encode contextual memories acquired close in time.}

(a) Miniscope methodology: GCaMP6f virus was injected into the RSC, and a Miniscope was used to image calcium transients in RSC neurons across repeated imaging sessions.

(b) GCaMP6f expression within the RSC is limited to specific sub-layers of the RSC. Scale: $1 \mathrm{~mm}$; inset, $100 \mu \mathrm{m}$.

(c) Example of maximum intensity projection of processed calcium signals during context exploration. Scale: $50 \mu \mathrm{m}$.

(d) Representative calcium traces from 15 putative RSC neurons from one mouse. Scale: 30 s.

(e) Overlapping RSC ensembles encode distinct memories acquired close in time. Top: Mice were imaged while exploring three novel contexts (A, $B$, and $C$ ) separated by 7 days or 5 hours. Bottom left: Overlapping neurons in RSC ensembles in a representative mouse when contexts were separated by 7 days (orange) and 5 hours (blue). Bottom right: RSC neuronal ensembles display greater overlap when contexts were separated by 5 hours (5h) vs. 7 days $(7 d ; n=12$ mice per group, paired t-test, $\mathrm{t}=4.8$ ).

(f) RSC ensemble firing rate is more correlated when two contexts are explored close in time. Left: RSC neuronal firing rate (active frames/s) for all neurons from one mouse during each context exploration ranked based on their normalized firing rate in context $\mathrm{C}$. High firing rate neurons continue to fire at high rates when contexts are explored close in time ( 5 hours vs. 7 days apart). Right: Population Vector Correlation (PVC) for normalized firing rates is higher when contexts are explored close in time (i.e., 5 hours vs 7 days apart; $n=9$ mice per group; paired ttest, $\mathrm{t}=3.2$ ).

(g) A Naïve Bayes (NB) classifier is better at distinguishing two contexts explored 7 days apart vs 5 hours apart. The AUC (area under the curve) for the binary NB classification, using neuronal activity between sessions, is higher for sessions recorded 7 days apart than sessions recorded 5 hours apart $(n=9$ mice per group; paired $t$-test, $t=3.5)$. Chance level performance of the classifier $(A \cup C=0.5)$ is represented by a dashed line. Spike probabilities were binned at 10 seconds in non-overlapping intervals.

(h) Representative neuronal coactivity across sessions: The stability of neuronal coactivity across sessions is represented as the absolute difference in pairwise correlations between sessions (i.e. Pairwise correlation for Session 2 - Pairwise correlation for Session 1). Absolute difference in coactivity when contexts are explored 7 days (left panel) or 5 hours (right panel) for 50 cell pairs. Higher numbers (darker color) indicate more stable coactivity patterns.

(i) Neuronal coactivity is more stable when contexts are explored close in time. The coactivity of neuronal pairs is more stable in two contexts explored $5 \mathrm{~h}$ vs $7 \mathrm{~d}$ apart $(\mathrm{n}=9$ mice per group; paired t-test, $\mathrm{t}=3.4$ ).

Data represent mean \pm s.e.m. and each data point, ${ }^{*} p<0.05,{ }^{* *} p<0.01,{ }^{* * *} p<0.001$. 
bioRxiv preprint doi: https://doi. org/10.1101/2021.1028.466343. this version posted December 7,2021 . The copyright holder for this preprint (which was not certified by peer review) is the author/funder, who has granted bioRxiv a license to display the preprint in perpetuity. It is made available under aCC-BY-ND 4.0 International license.

\section{A}
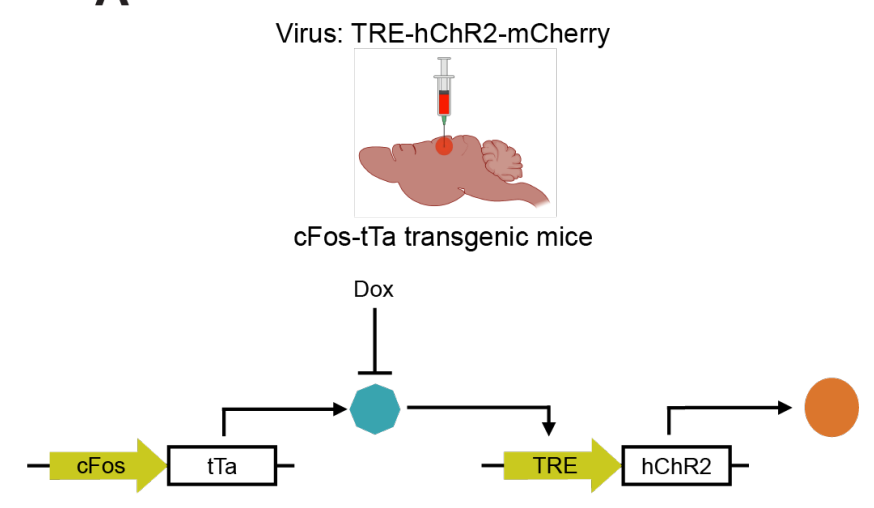

B

Control

TTA-ChR2
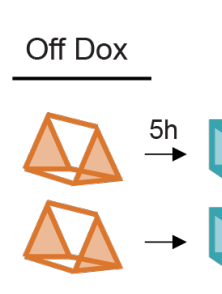

A

B
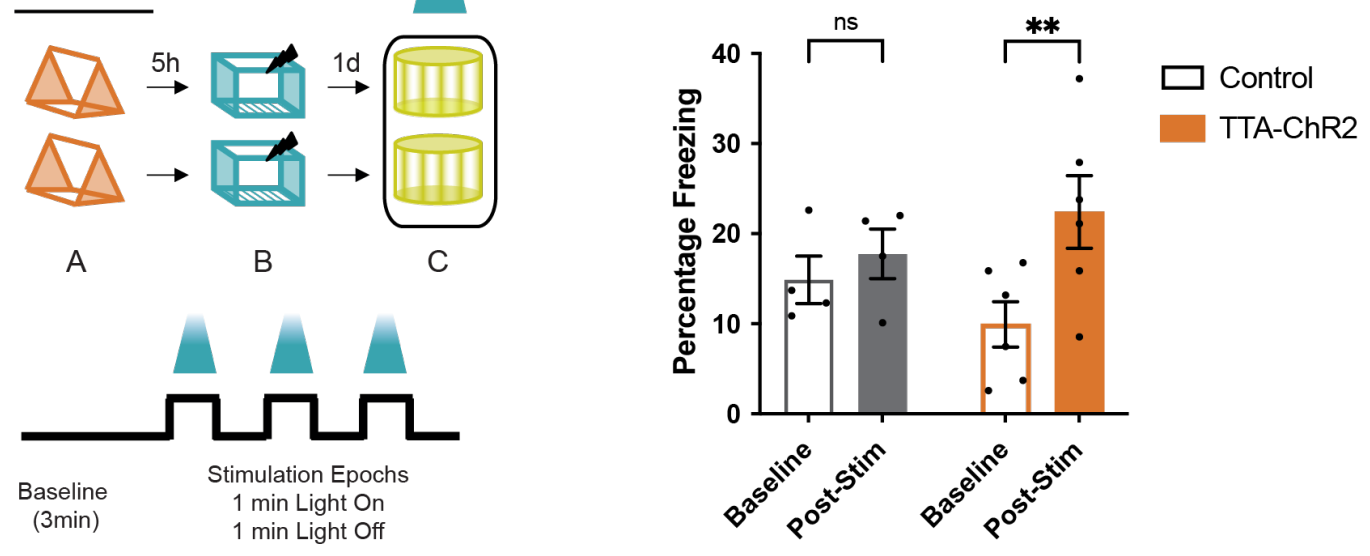

C

Off Dox

Control

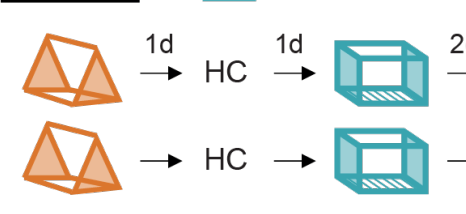

A

B

$\stackrel{2 \mathrm{~d}}{\rightarrow}$
$\rightarrow$

Imm
Shock $\stackrel{1 d}{\rightarrow}$

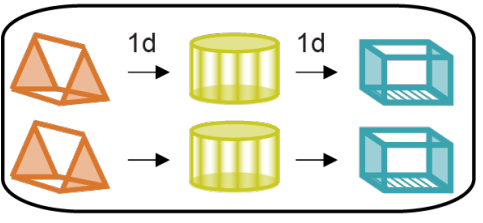

Test A
Test

Novel
Test B
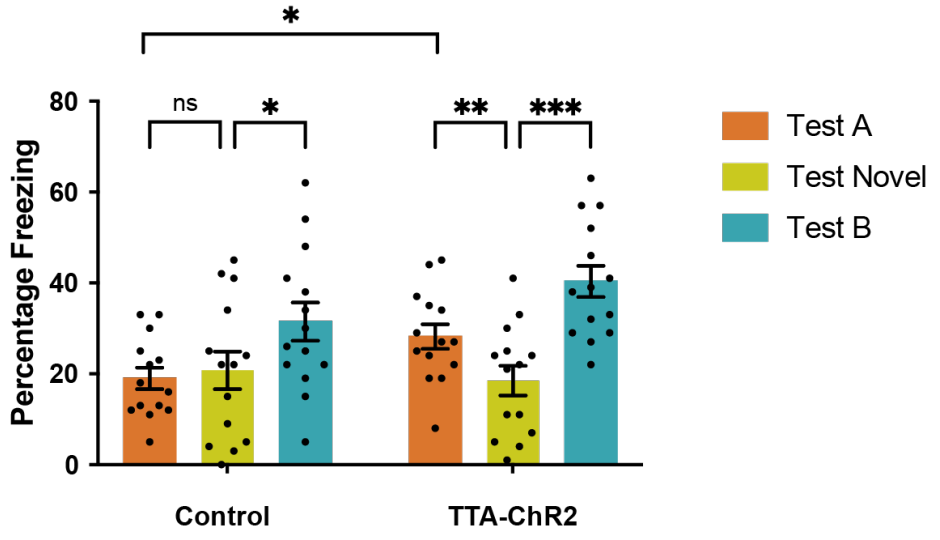


\section{Figure 2. Overlap in RSC neuronal ensembles is sufficient to link contextual memories.}

(a) Schematic of the TetTag system: cFos-tTa or wildtype littermate mice were injected with the TRE-hChR2-mCherry virus. In the absence of doxycycline, the activation of the cFos promoter allows expression of Channelrhodopsin (hChR2).

(b) Optogenetic reactivation of an RSC ensemble underlying a linked memory is sufficient for fear expression: Top left: RSC ensemble activated during the exploration of the first context (context A) was tagged. Five hours later, the second context (context B) was paired with a footshock. The following day, mice were tested in a third novel context (context $\mathrm{C}$ ) with optogenetic reactivation of the tagged (Context A) RSC neuronal ensemble. Bottom left: Optogenetic stimulation (473nm laser, $5 \mathrm{~ms}$ pulses, $5 \mathrm{~Hz}$ ) was preceded by $3 \mathrm{~min}$ of context acclimation, followed by three 1minute trials of light pulses, separated by a 1-minute period of light off. Right: Reactivation of the RSC neuronal ensemble tagged during the linked context exploration (context $A$ ) increases freezing in cFos-tTa mice during the post-stimulation period, while the freezing in the control group remains unchanged (Two-way RM ANOVA, $F_{\text {Interaction }}(1,8)=5.4, p<0.05$; Sidak's multiple comparisons test).

(c) Reactivation of the RSC ensemble underlying the first context memory (context A) extends the temporal window for memory linking: Mice were allowed to explore 2 contexts (context $A$ and B) separated by 2 days (a time interval when memories are not linked). On the day between the two context exposures, the RSC ensemble tagged during the first context visited (context A) was reactivated optogenetically. While control mice do not link the 2 contexts, reactivation of the first context ensemble leads to robust contextual memory linking: freezing in both previously explored contexts (Context $A$ : linked context and Context $B$ : shock context) is higher than the freezing in a novel context. The control group freeze similarly in context $A$ and novel context, but the freezing in context $B$ (shock context) is higher than freezing in a novel context (Two-way RM ANOVA, $F_{\text {GroupXContext }}(2,52)=3.3, p<0.04$; Dunnett's multiple comparisons test for contexts and Uncorrected Fisher's LSD for groups).

Data represent mean \pm s.e.m. and each data point, ${ }^{*} p<0.05,{ }^{* *} p<0.01,{ }^{* * *} p<0.001$. 
A

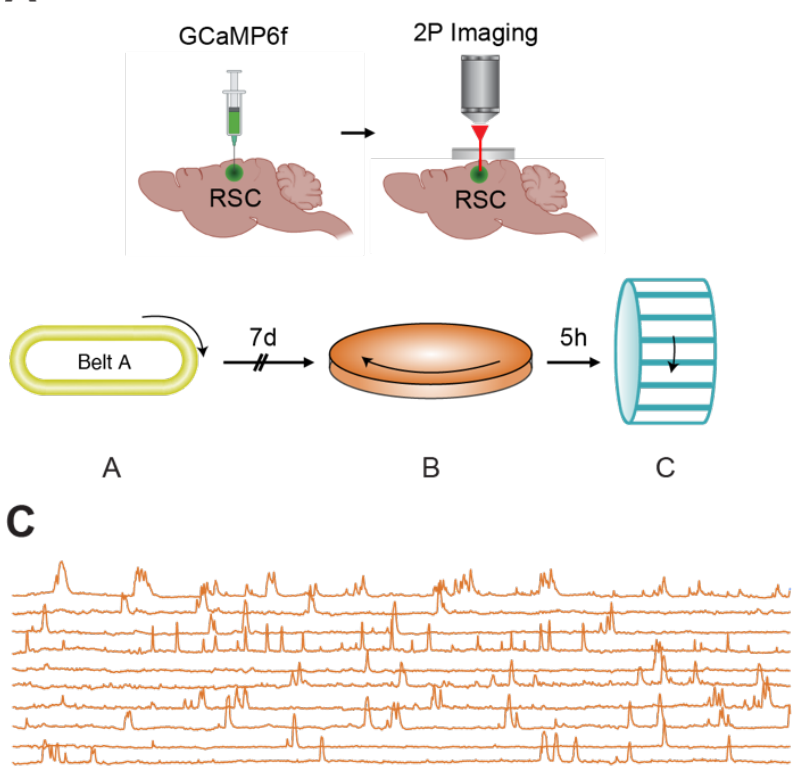

D

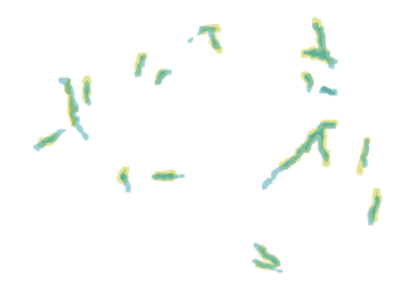

Overlapping segments in $\mathrm{A}$ and $\mathrm{C}$ $7 \mathrm{~d}$ apart
B

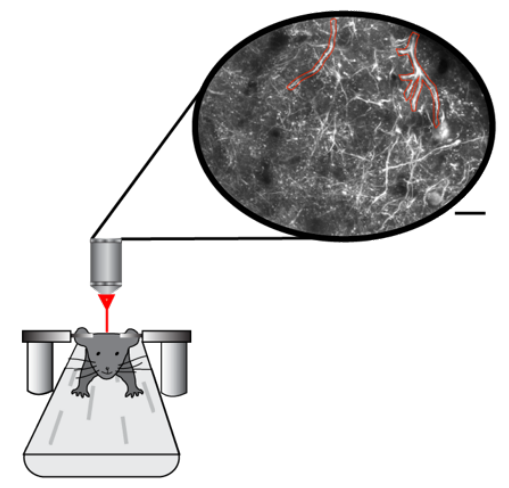

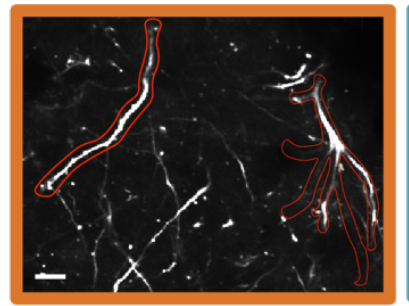

Context B

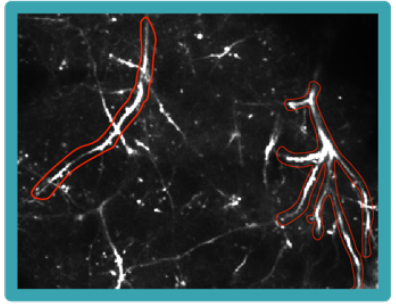

Context C
$\mathbf{E}$

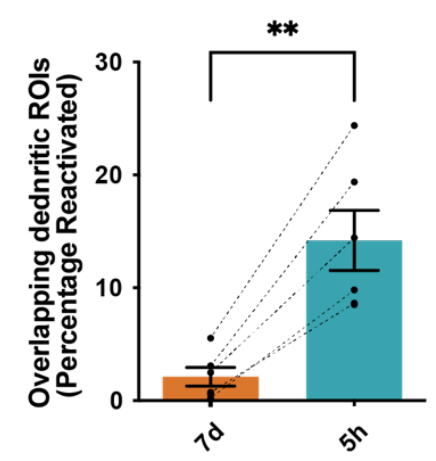

Figure 3. Overlapping dendritic segments encode memories of two contexts explored close in time.

(a) Experimental setup: A $3 \mathrm{~mm}$ square craniotomy was made over the RSC spanning the midline, and GCaMP6f was injected bilaterally into the RSC. During imaging sessions, mice were headfixed and experienced three novel contexts either 7 days or 5 hours apart as dendritic calcium transients were imaged using a two-photon microscope. Contexts comprised distinct visual, auditory, olfactory, and tactile cues (figure S8).

(b) Representative images of the same dendritic segments being tracked across 7 days. Top: Maximum intensity projection from one imaging session showing apical dendritic segments activated during the context. Two different dendritic segments are outlined in red. Scale: $20 \mu \mathrm{m}$. Bottom: Dendritic segments from top panel tracked across two imaging sessions 5 hours apart. Scale: $10 \mu \mathrm{m}$.

(c) Representative calcium traces from 10 putative RSC dendritic segments. Scale: 2 min. 
bioRxiv preprint doi: https://doi.org/10.1101/2021.10.28.466343; this version posted December 7, 2021. The copyright holder for this preprint

(which was not certified by peer review) is the author/funder, who has granted bioRxiv a license to display the preprint in perpetuity. It is made available under aCC-BY-ND 4.0 International license.

(d) Overlapping dendritic segments reactivated when contexts are separated by 7 days (left) or 5 hours (right) from one mouse.

(e) The same dendritic segments are more likely to be activated when context exposures are 5 hours (5h) apart vs 7 days (7d) apart. (Paired t-test; $\mathrm{t}=6.47 ; p<0.01 ; \mathrm{n}=6$ mice).

Data represent mean \pm s.e.m. and each data point. 
A

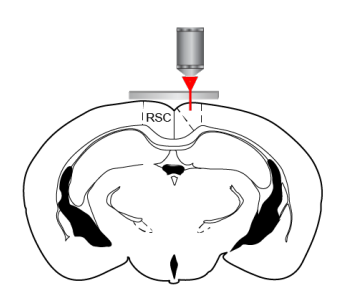

Thy1-YFP mice
B

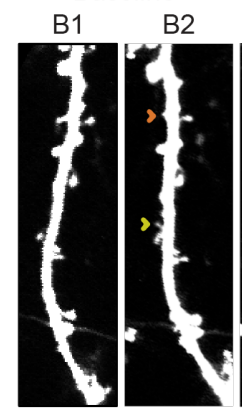

D

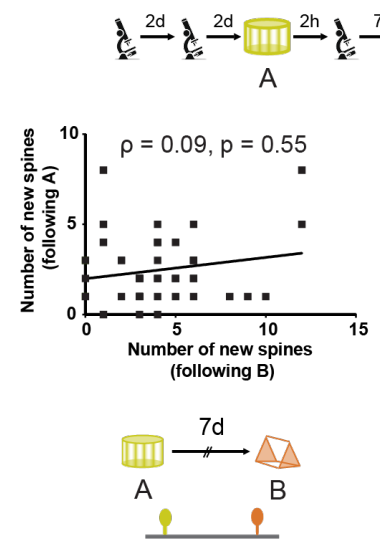

$\mathbf{F}$

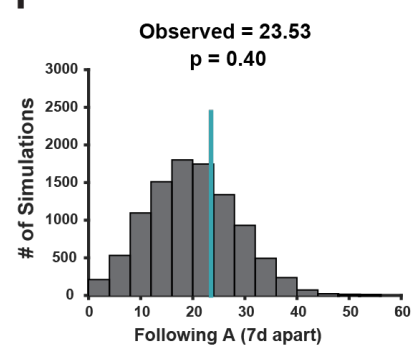

Percent of Clustered Spines on $\mathrm{C}$ dendrites

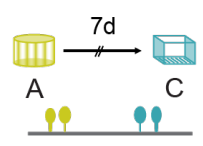

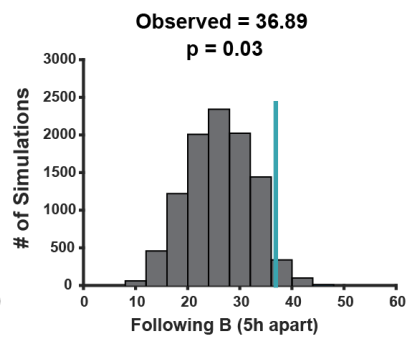

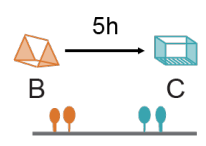

Context exposure

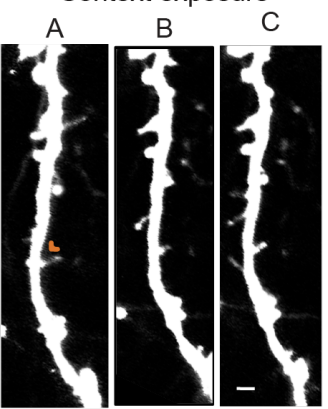

C

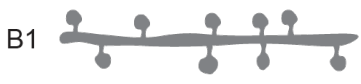

B2

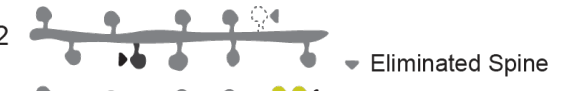

A

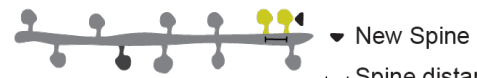

B

- Spine distance $<5$ um

C

E

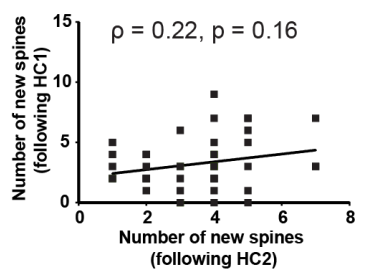

$\mathrm{HC} 1 \stackrel{7 \mathrm{~d}}{\longrightarrow} \mathrm{HC} 2$

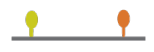

G
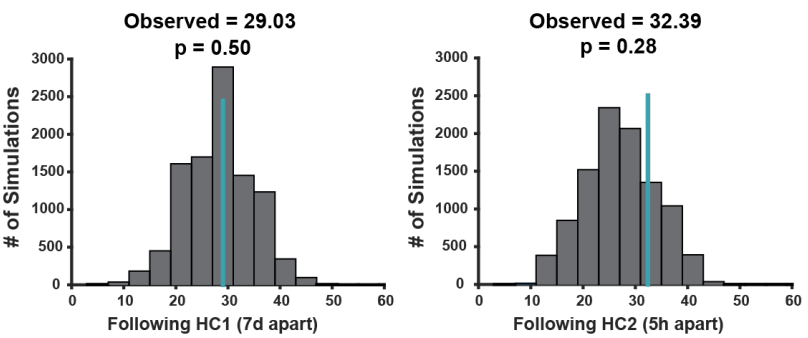

Percent of Clustered Spines on HC3 dendrites

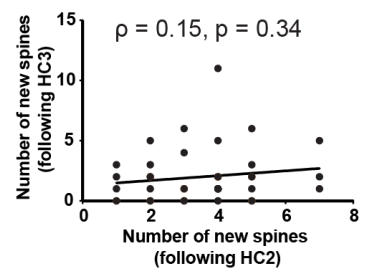

$\mathrm{HC} 2 \stackrel{5 \mathrm{~h}}{\longrightarrow} \mathrm{HC} 3$

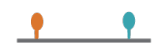
$\mathrm{HC} 2 \stackrel{5 \mathrm{~h}}{\longrightarrow} \mathrm{HC} 3$

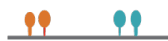

Figure 4. Overlapping dendritic segments gain spine clusters following memory linking.

(a) Experimental setup: A $3 \mathrm{~mm}$ square craniotomy was made over the RSC of Thy1-YFP mice. Apical RSC dendrites were imaged to measure context exposure and memory linking related spine dynamics. 
(b) Representative example of spine dynamics during longitudinal imaging. Lost spine: orange arrowhead. Gained spine: yellow arrowhead. B1 and B2: Baseline imaging sessions; A, B and C: Exposure to contexts $A, B$ and $C$ respectively. Scale: $1 \mu \mathrm{m}$.

(c) Schematic of various spine dynamics (spine addition, elimination, and clustering) measured.

(d) New spines are likely to be added to the same dendritic segments when contexts are explored close in time. Left: Number of new spines added to a dendritic segment following Context $A$ and B exposure ( 7 days apart) are not correlated $(\rho=0.09, p=0.55)$. Right: Number of new spines added to a dendritic segment following Context $B$ and $C$ exposure ( 5 hours apart) are correlated $(\rho=0.37, p=0.01)$. Spearman's correlation was used.

(e) For home cages $(\mathrm{HC})$ controls, the number of new spines added to a dendritic segment are not correlated whether imaging sessions are separated by either 7 days (right, $\rho=0.22, p=0.16$ ) or 5 hours (left, $\rho=0.15, p=0.34$ ). Spearman's correlation was used.

(f) Co-allocation of clustered spines to dendritic segments: The probability that clustered spines are co-allocated to the same dendritic segment by chance following two imaging sessions was calculated by randomly distributing clustered spine position following one imaging session. Left: The percentage of clustered spines co-allocated to the same dendritic segments following contexts $A$ and $C$ (explored 7 days apart) are at chance levels. Right: In contrast, the percentage of clustered spines co-allocated to the same dendritic segments following contexts $\mathrm{B}$ and $\mathrm{C}$ (explored 5 hours apart) are more than expected by chance. $n=6$ mice; 10000 permutations.

(g) For home cages ( $\mathrm{HC})$ controls, the percentage of clustered spines that were added to segments also containing clustered spines in a previous imaging session (7 days or 5 hours prior) were at chance levels. $n=5$ mice; 10000 permutations. 


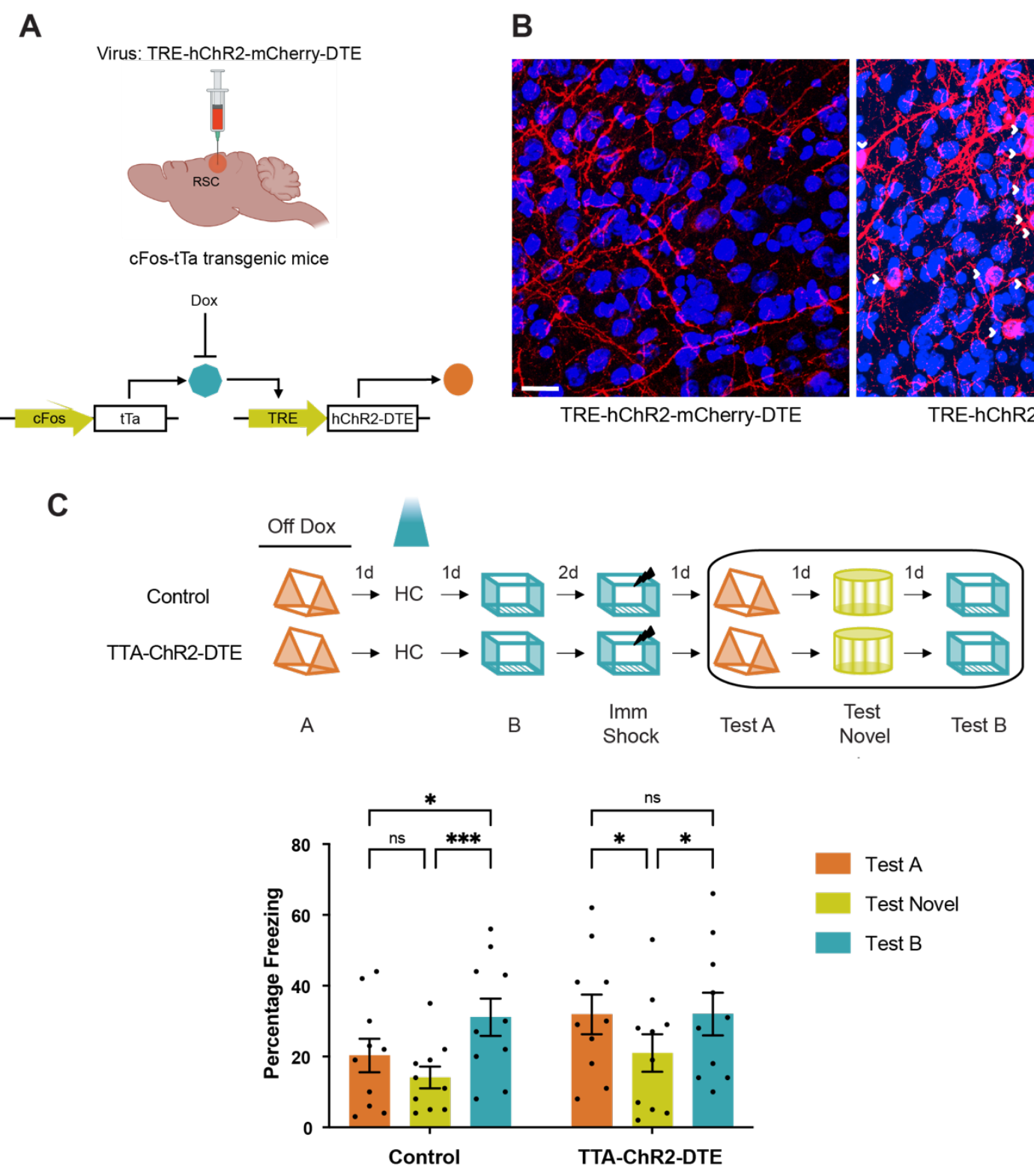

Figure 5. Optogenetic reactivation of RSC dendritic ensembles links contextual memories

(a) The TRE-hChr2-mCherry-DTE virus was designed to optogenetically reactivate previously activated dendritic segments. The key genetic elements include 1) TRE promoter and 2) Dendritic Targeting Element (DTE) at the 5' end: The DTE element targets mRNA to activated dendrites and has been used to target photoactivatable molecules to activated dendrites/spines (8). TREhChr2-mCherry-DTE virus was injected into cFos-tTa mice to express Channelrhodopsin in the recently activated dendritic segments of cFos-expressing neurons.

(b) Representative RSC images of cFos-tTa mice injected with TRE-hChR2-mCherry-DTE (left) and TRE-hChR2-mCherry (right) showing selective expression of Channelrhodopsin in dendritic 
segments in the presence of DTE. White arrowheads: somatic expression of hChR2 in the absence of DTE. Scale: $20 \mu \mathrm{m}$

(c) Experimental setup: Top: Mice explored two contexts 2 days apart (a time interval when memories are not linked). On the day between the 2 context exposures, the dendrites activated during the first context exposure were reactivated optogenetically. Bottom: Reactivation of context $A$ dendrites, on the day between the two context exposures (contexts $A$ and $B$ ), results in high freezing in both the previously explored contexts (context A: linked context and context B: shock context) relative to freezing in a novel context. The control mice freeze similarly in context $A$ and novel context, but the freezing in context $B$ (shock context) is higher than freezing in context $A$ or a novel context (Two-way RM ANOVA, $F_{\text {time }}(2,18)=9.3, P=0.001$; Dunnett's multiple comparisons test). Data represent mean \pm s.e.m. and each data point, ${ }^{*} p<0.05,{ }^{* *} p<0.01$, ${ }^{* * *}$ $p<0.0001$. 


\section{Supplementary figures}
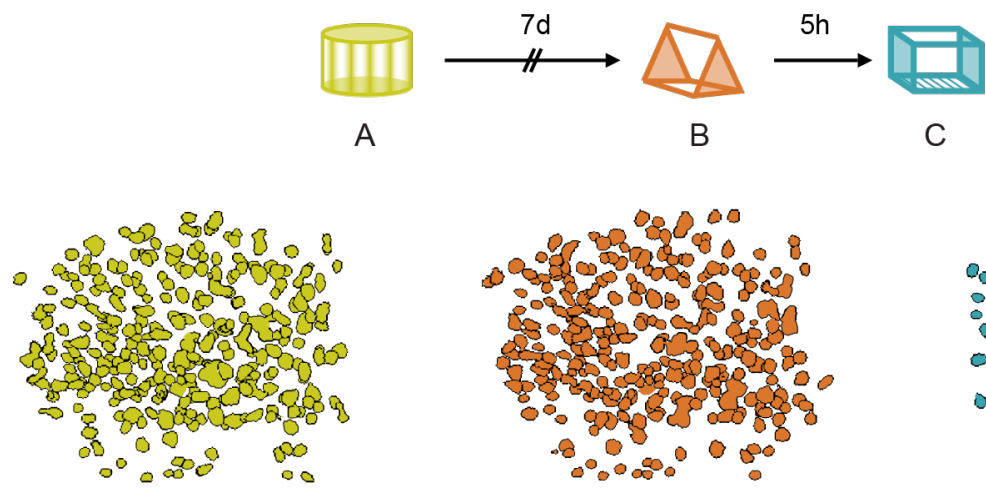

Context A

Context B

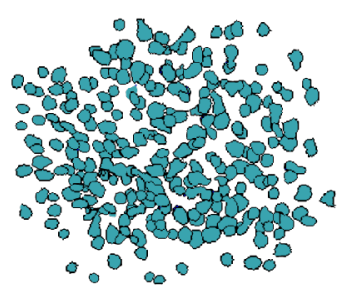

Context C

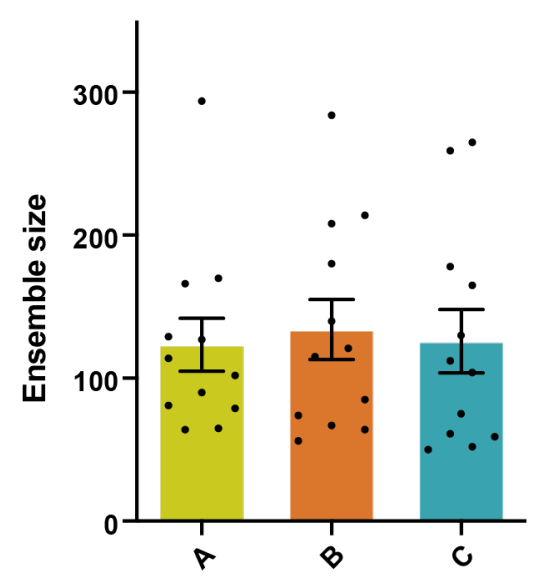

Figure S1. RSC ensemble size remains stable across multiple imaging sessions. RSC ensemble size captured by Miniscope imaging remains stable across hours and days when different contexts are imaged (4599 putative RSC neurons, $132.9 \pm 11.6$ neurons per session, One-way repeated measures ANOVA, $\mathrm{F}(1.08,11.9)=0.52, p=0.5, \mathrm{n}=12$ mice per group). Please note that although the size of these ensembles remains unchanged, the neurons participating in these ensembles may change. Data represent mean \pm s.e.m. and each data point. 
A
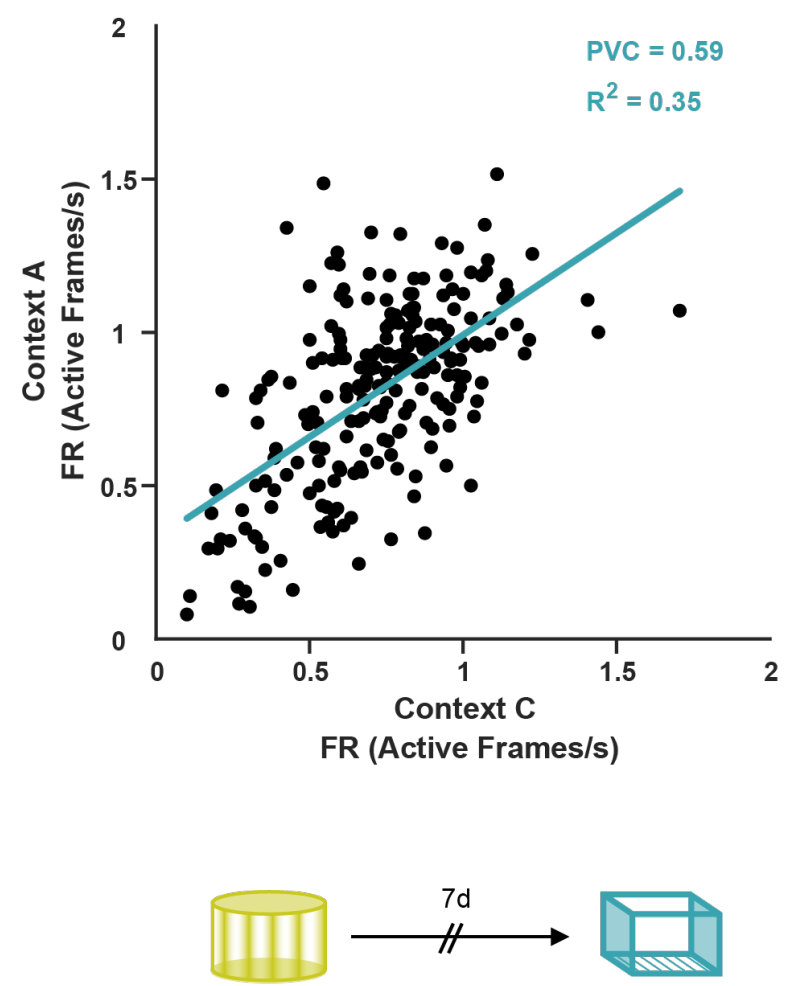

A

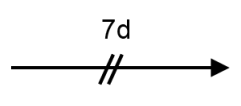

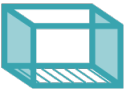

C
B
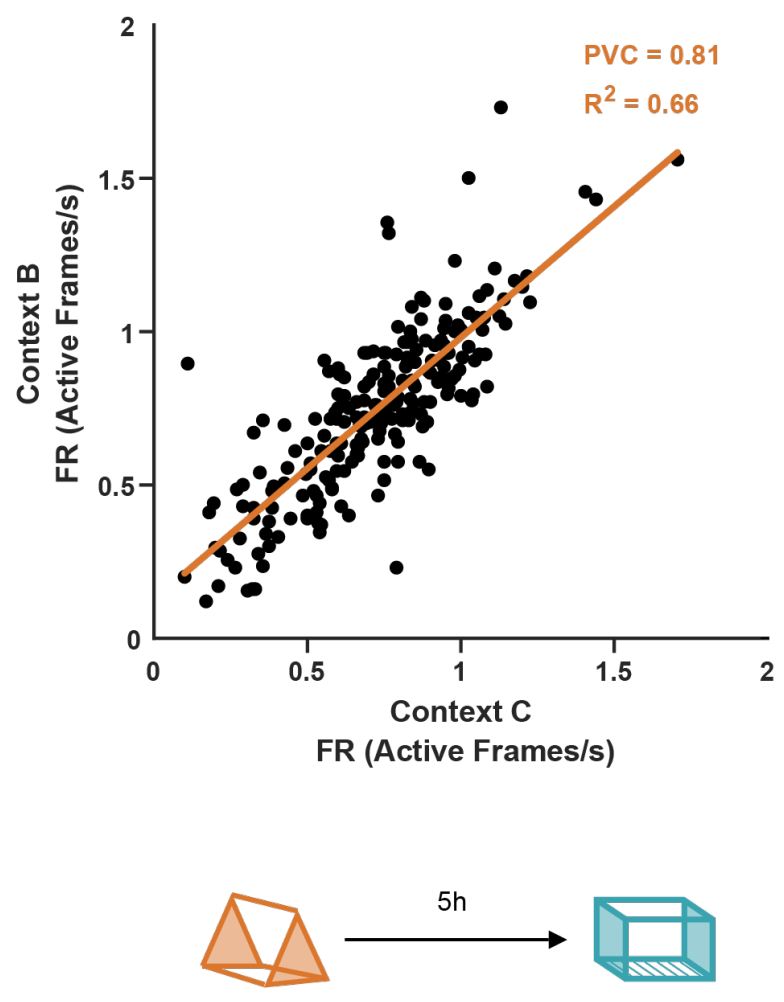

C

Figure S2. Neuronal firing rate is more correlated when two contexts are explored closer in time. Scatter plot of firing rate of all neurons from one mouse in context $A$ ( $a, 7$ days apart) and context $B$ (b, 5 hours apart) as a function of firing rate in context $C$ highlights that neuronal firing rate is maintained when two contexts are explored close in time. Lines represent least-squares linear regression. 


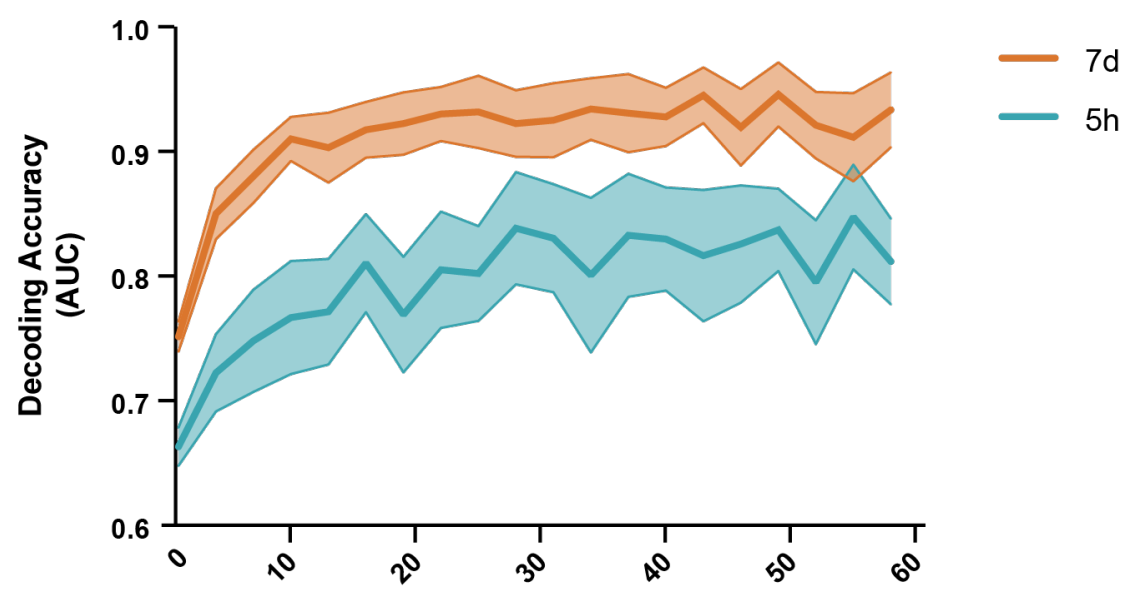

BIn Size (s)

Figure S3. Naïve Bayes (NB) classifier is more accurate at distinguishing imaging sessions recorded 7 days vs 5 hours apart irrespective of bin size. AUC (area under the curve) for the binary NB classification between sessions recorded 7 days (7d) apart or 5 hours (5h) apart using neuronal activity indicates that neuronal activity can be used to distinguish between contexts explored 7 days apart more accurately than contexts explored 5 hours apart. Spike probabilities were binned for non-overlapping intervals ranging from 0.5 to 60 seconds (step size $0.5 \mathrm{~s}$; Twoway Repeated Measures ANOVA for AUC by bin size; $F_{\text {Group }}(1,16)=6.2, p<0.05, \mathrm{n}=9$ mice per group). Data represent mean \pm s.e.m. Chance Levels performance of the $A \cup C=0.5$. 
A

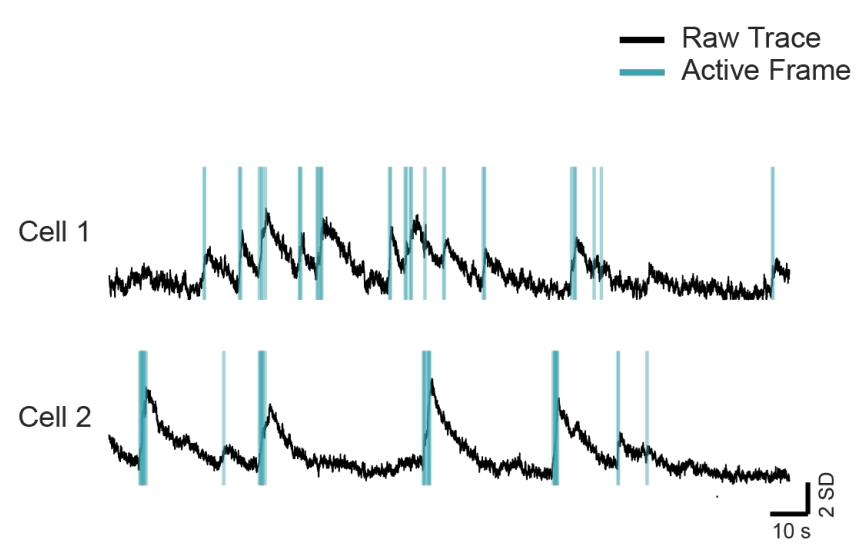

B
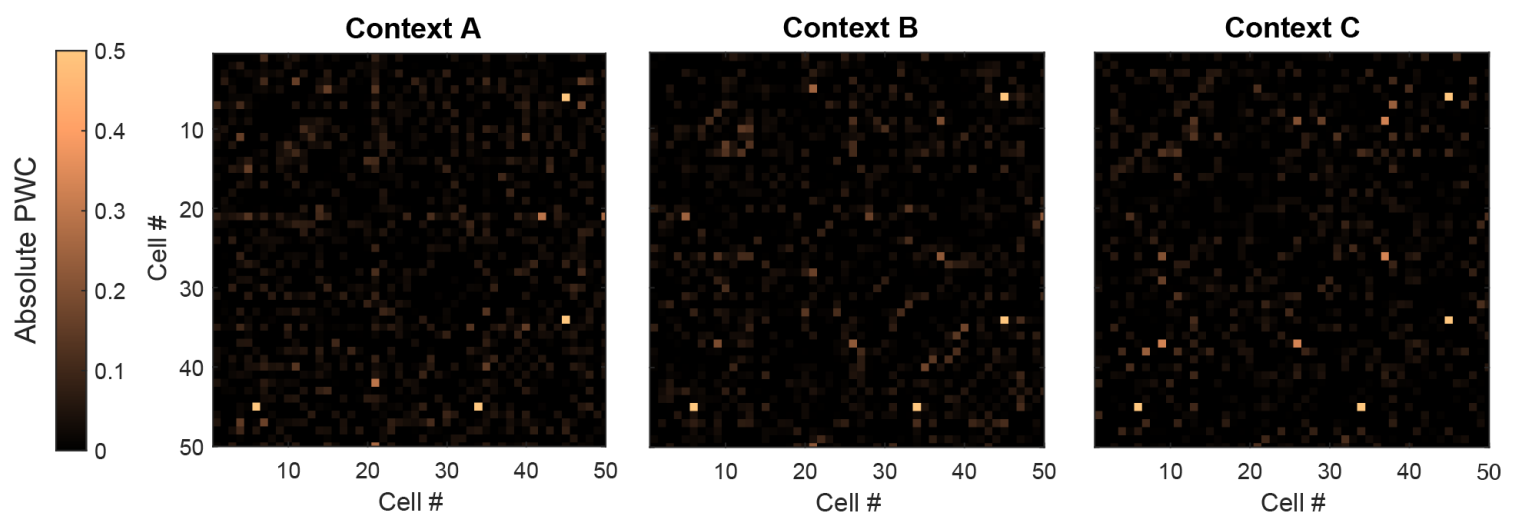

C

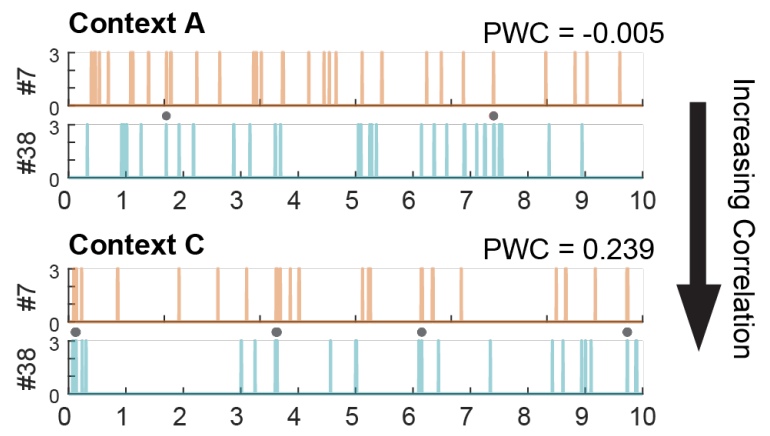

- Frame with simultaneous activity in both cells
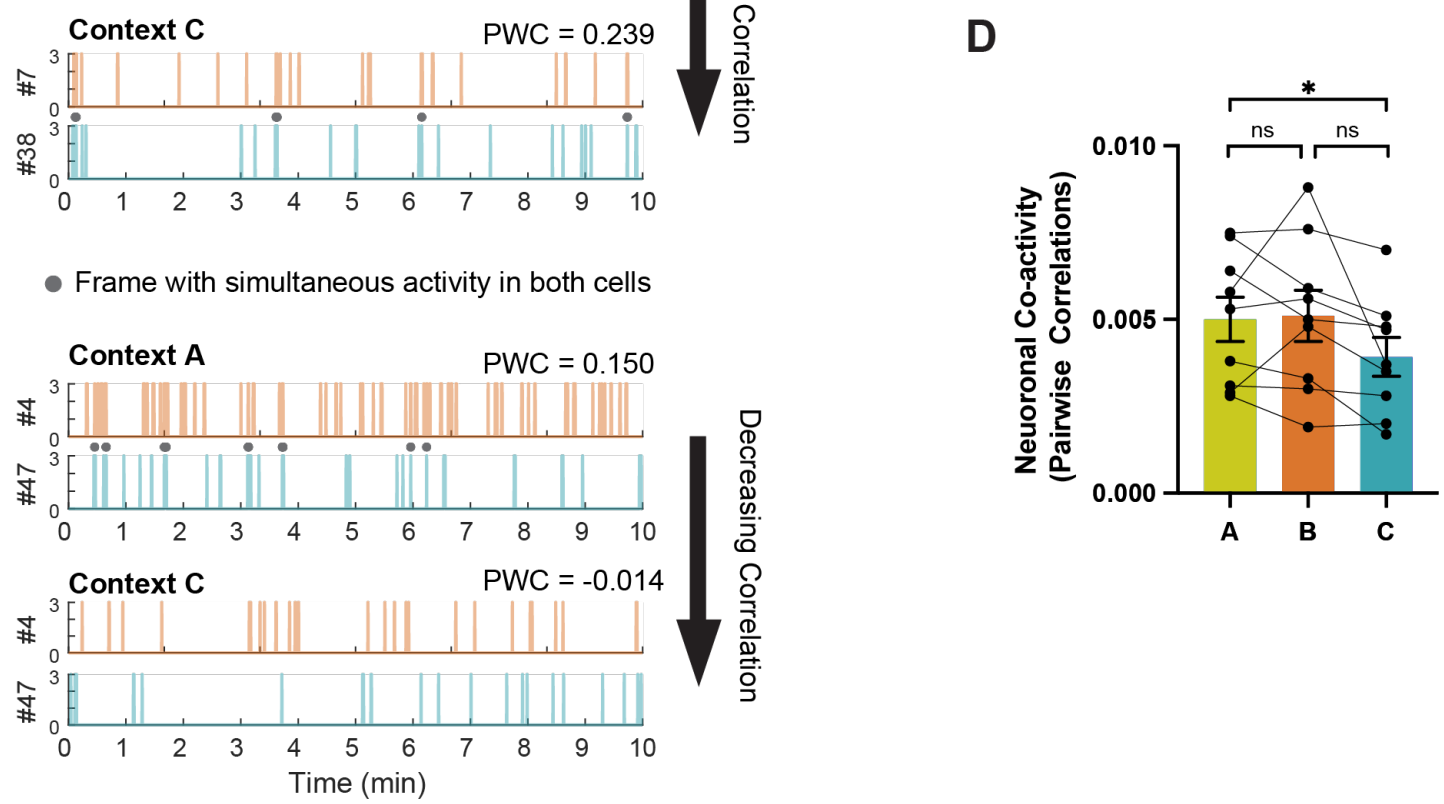


\section{Figure S4. Coactivity among RSC neurons during multiple contextual exposures}

(a) Example of two co-active neurons: For each imaging session, deconvolved neuronal activity was binned into 100ms periods, and the Pairwise correlation (PWC: Pearson correlation of the number of active frames) for each neuron pair was calculated. Black: raw calcium trace and Blue: frames identified as active following deconvolution of the calcium trace.

(b) Pairwise Correlation (PWC) map for each context for 50 cell pairs from one mouse. Absolute Pearson's correlation coefficients were plotted.

(c) Top Panel: Example of a neuronal pair with increasing PWC between imaging sessions: Cell pair (7 and 38: arbitrary cell IDs) display more coactivity (number of simultaneous active frames) during Context $\mathrm{C}$ exploration than Context A exploration. Bottom Panel: Example of a neuronal pair with decreasing PWC between imaging sessions: Cell pair (4 and 47: arbitrary cell IDs) display less coactivity during Context $\mathrm{C}$ exploration than Context $\mathrm{A}$ exploration.

(d) Average PWCs across the three context exposures display a small decrease (One-way repeated measure ANOVA; $\mathrm{F}(1.6,12.9)=4.05, p=0.05$; Tukey's post-hoc test, $\mathrm{n}=9$ mice per group). Data represent mean \pm s.e.m. and each data point, ${ }^{*} p<0.05$. 
A

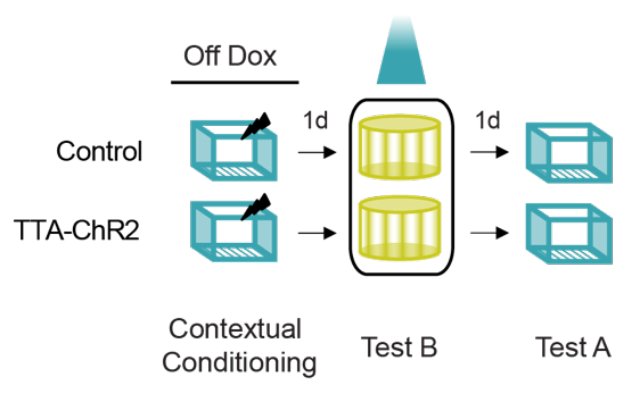

C

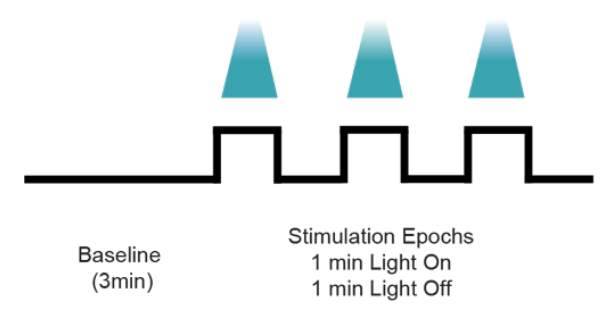

$\mathbf{F}$

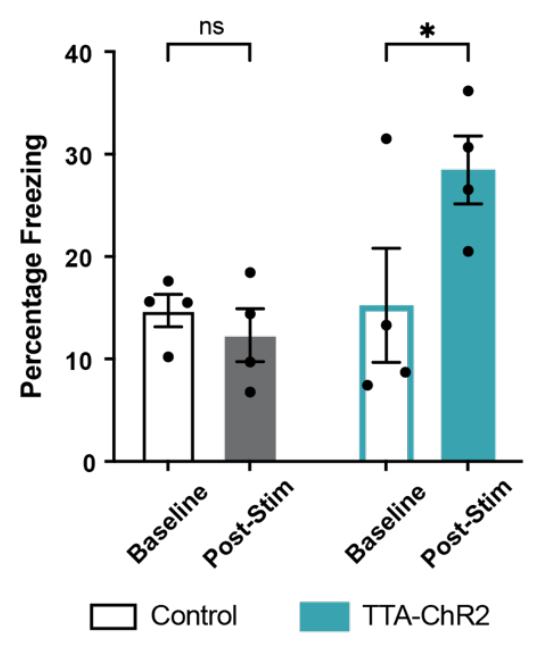

Test B

B

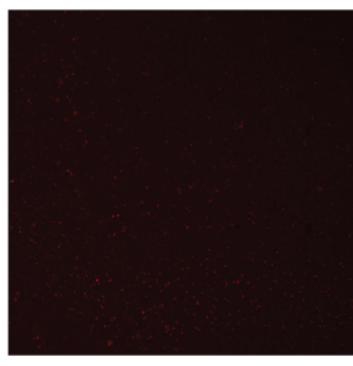

Control

D

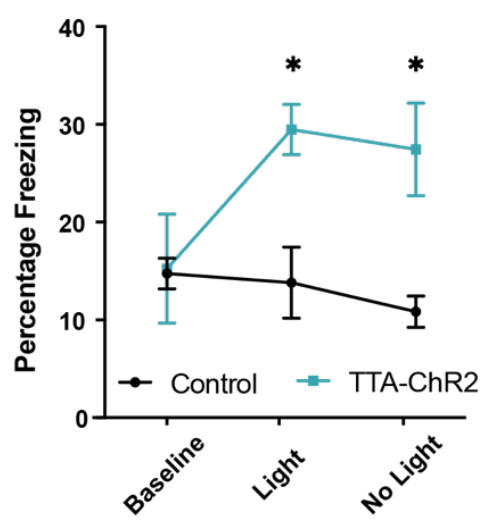

Test B

G

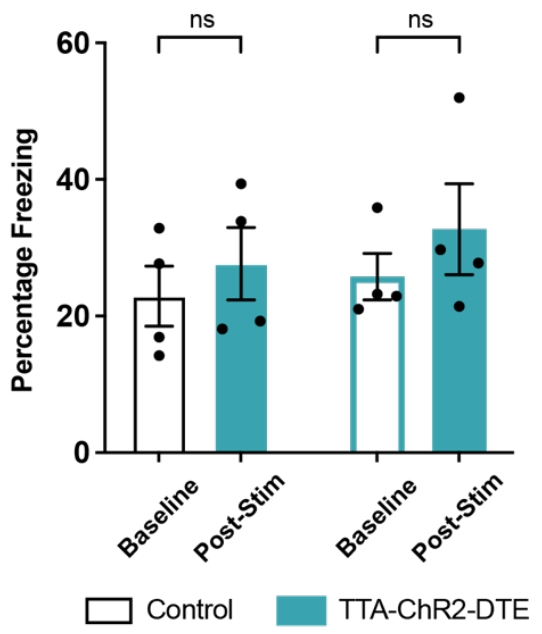

Test B

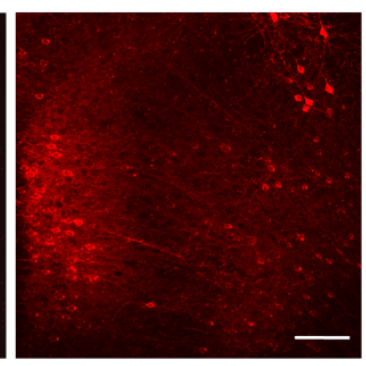

TTA-ChR2

E

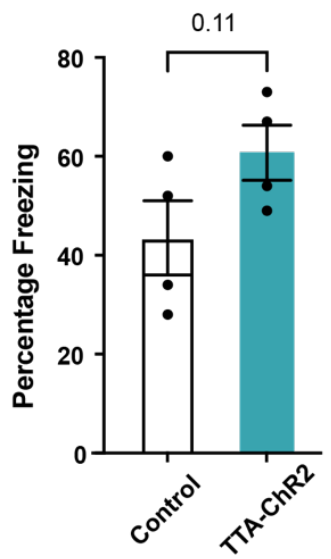

Test A

H

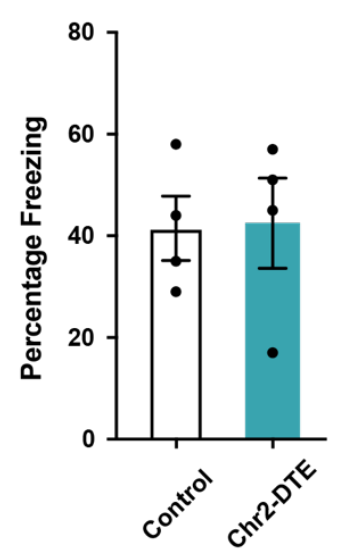

Test A 
Figure S5. RSC neuronal ensemble (and not dendritic ensemble reactivation) following contextual fear conditioning results in fear expression

(a) Experimental setup: cFos-tTa (TTA-Chr2) mice and their wildtype littermates (Control) underwent bilateral viral injections (TRE-ChR2-mCherry) and optic cannula implants. Mice were taken off doxycycline chow (three days before contextual fear conditioning in context A: 2 footshocks, 2s, 0.7mA) to allow c-fos promoter driven tTA and Channelrhodopsin (ChR2) expression. Following contextual fear conditioning, mice were tested in a novel context (Test $B$ ) while the previously tagged neurons were activated. The following day mice were retested without any optogenetic manipulation in the training context (Test $A$ ).

(b) ChR2-mcherry expression in the RSC one day after fear learning. Scale: $100 \mu \mathrm{m}$.

(c) Optogenetic stimulation protocol: same as described for figure $2 \mathrm{~b}$.

(d) During Test B, TTA-Chr2 mice and their wildtype littermates display similar freezing levels during the baseline period. Upon reactivation of the RSC neuronal ensemble tagged during contextual fear conditioning, TTA-Chr2 mice display more freezing compared to the control group during the post-baseline stimulation as well as non-stimulation epochs (Two-way RM ANOVA, group X time interaction, $\mathrm{F}(2,12)=6.95, p<0.01$, Uncorrected Fisher's LSD, $p<0.05)$.

(e) During Test A, the TTA-Chr2 mice display comparable freezing to the control mice $(\mathrm{t}=1.85$, $\mathrm{df}=6, p=0.11$ ).

(f) Same data as in (d): Freezing during the two post-stimulation conditions (with laser and without laser stimulation) was not different. Therefore, freezing during this period is presented together as post-stimulation freezing (Two-way RM ANOVA, group $X$ time interaction, $F(1,6)=11.93, p$ $<0.01$, Sidak's post hoc tests).

(g) Reactivation of previously activated dendrites is not sufficient for fear memory expression: Experimental set up is the same as (a) but animals were injected with TRE-hChR2-mCherry-DTE or TRE-mCherry-DTE virus in the RSC to reactivate dendritic segments active during contextual fear conditioning. Both groups display similar freezing during baseline and post-stimulation epochs during testing in a novel context (Two-way RM ANOVA, group X time interaction, $F(1,6)$ $=0.26, p=0.6$, Sidak's post hoc tests).

(h) Both groups (injected with TRE-hChR2-mCherry-DTE or TRE-mCherry-DTE virus in the RSC) display similar freezing during test in Context $\mathrm{A}(\mathrm{t}=0.09$, $\mathrm{df}=6, p=0.9)$.

Data represent mean \pm s.e.m. and each data point, ${ }^{*} p<0.05$, ns $=$ not significant. 
A

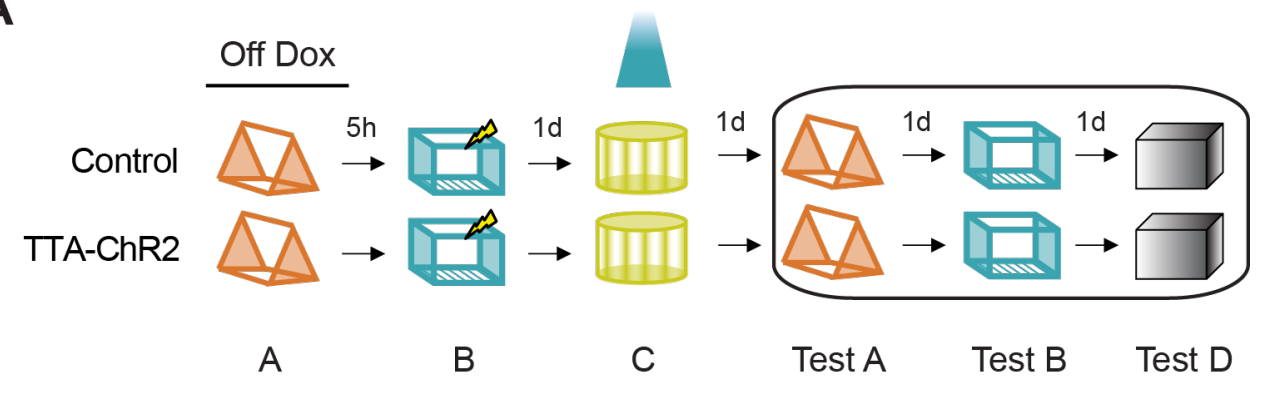

B

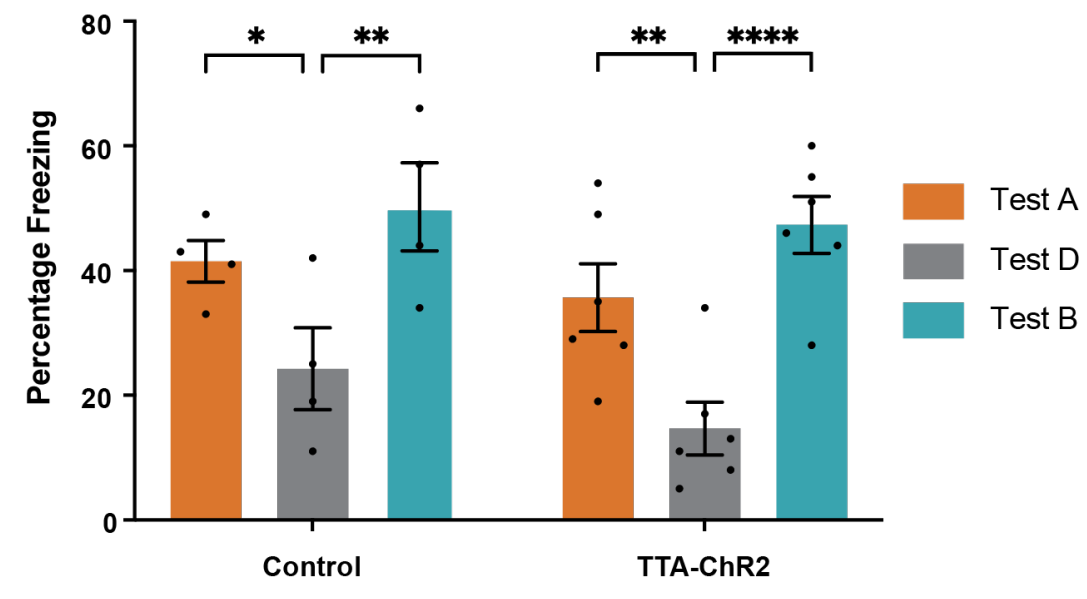

Figure S6. Fear expression due to reactivation of the linked memory ensemble is not driven by changes in the strength of memory linking.

(a) Following optogenetic activation of the first memory ensemble (figure 2b), mice were tested in the linked context (Context A), shock context (Context B), and in another novel context.

(b) Both groups of mice display robust memory linking, such that the freezing in both context $A$ (linked) and context B (shock) is higher than freezing in a novel context (Two-way RM ANOVA, $\mathrm{F}_{\text {time }}(2,16)=25.5, \mathrm{P}<0.0001$; Tukey's multiple comparisons test).

Data represent mean \pm s.e.m. and each data point, ${ }^{*} p<0.05,{ }^{* *} p<0.01,{ }^{* * *} p<0.0001$. 
B

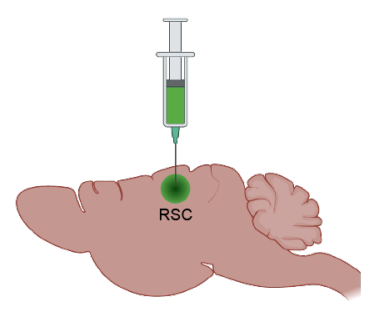

\section{Saline-CNO}

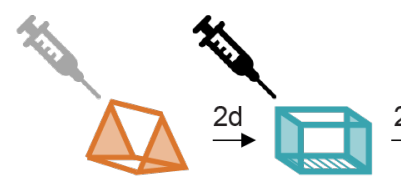

CNO-CNO
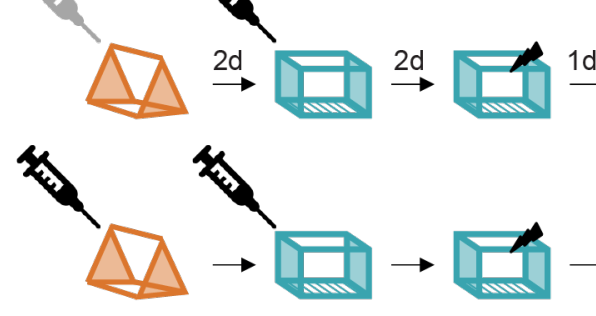

$\stackrel{1 d}{\rightarrow}$

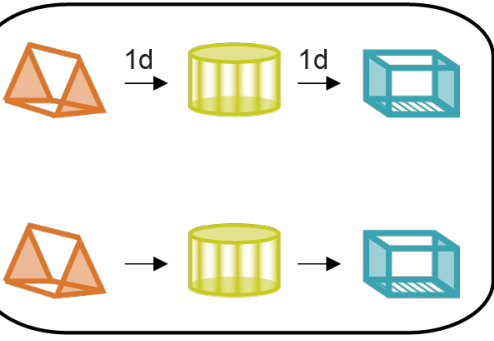

A

B $\quad \begin{aligned} & \text { Imm } \\ & \text { Shock }\end{aligned}$

$\begin{array}{cc} & \text { Test } \\ \text { Test A } & \text { Novel }\end{array}$

Test B

C

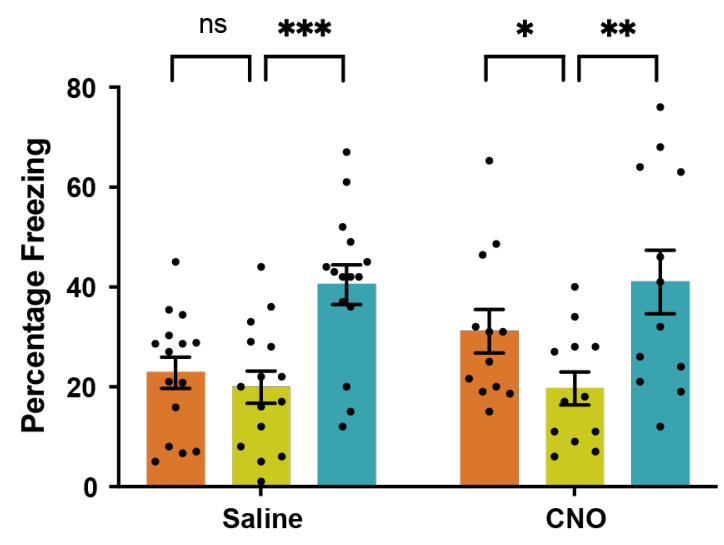

Test A

Test Novel

Test B

Figure S7. Chemogenetic manipulation of the neuronal ensemble overlap is sufficient to link two distinct contextual memories

(a) All mice received a bilateral injection of lentivirus DREADD hM3Dq.T2A.EGFP which infects a sparse population of RSC neurons.

(b) All mice explored two different contexts 2 days apart and were subsequently shocked in one of these contexts. Neuronal excitability was increased in a small subset of RSC neurons by 
administering a CNO $(0.5 \mathrm{mg} / \mathrm{kg})$ injection 45 mins before each context exploration. The control mice only received the $\mathrm{CNO}$ injection before the second context exploration.

(c) Control mice display low levels of freezing in a novel as well as the previously explored neutral (Context A) context but freeze more in the training context (Context A). In contrast, mice from the experimental group display memory linking: Both the previously explored contexts (Context $A$ and $B)$ elicit high freezing relative to the freezing in a novel context. (Two-way RM ANOVA, $F_{\text {time }}$ (1.8, $44.9)=28.45, P<0.0001$, Dunnett's multiple comparisons test).

Data represent mean \pm s.e.m. and each data point, ${ }^{*} p<0.05,{ }^{* *} p<0.01,{ }^{* * *} p<0.001$. 


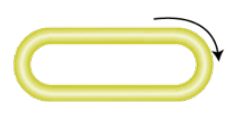

A

Visual Cues

Contextual Cues

Auditory \& Olfactory Cue
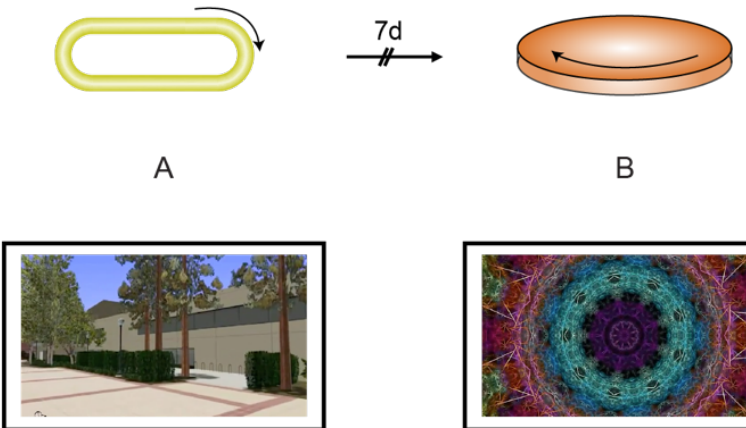

$\stackrel{5 \mathrm{~h}}{\longrightarrow}$

B
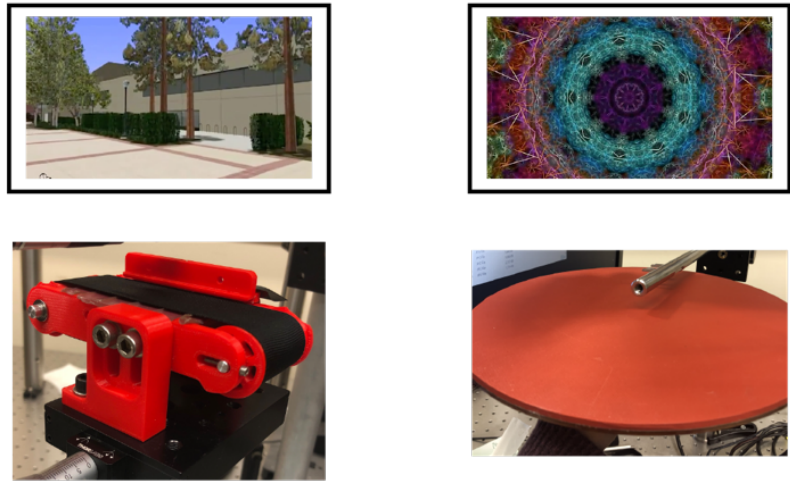

Simple Green

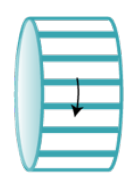

C
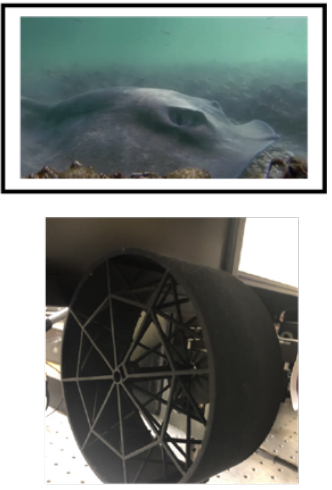

Acetic Acid

Figure S8. Experimental setup for functional dendritic co-allocation studies: Schematics of 3 distinct contexts (different auditory, visual, and olfactory cues as well as running apparatus) used in the head fixed experiments. Mice were exposed to 3 distinct contexts 7 days or 5 hours apart in a counterbalanced manner while RSC dendritic transients were imaged. 
A

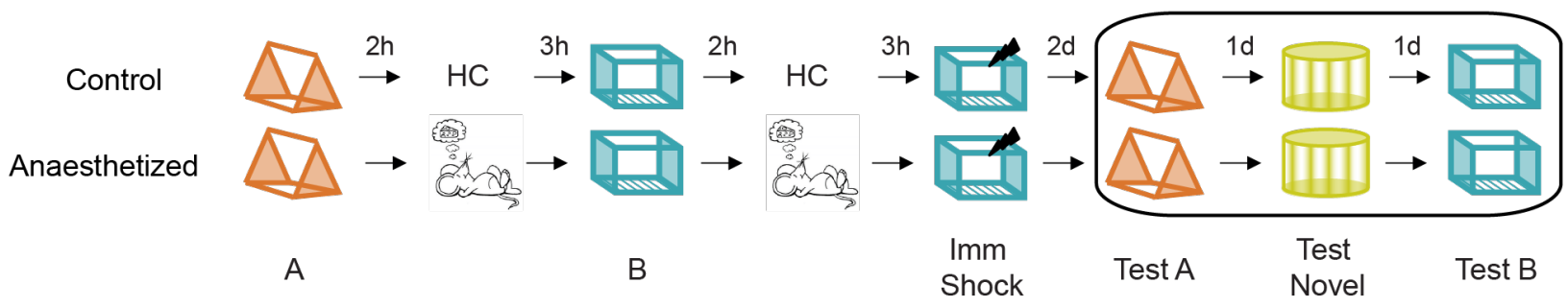

B

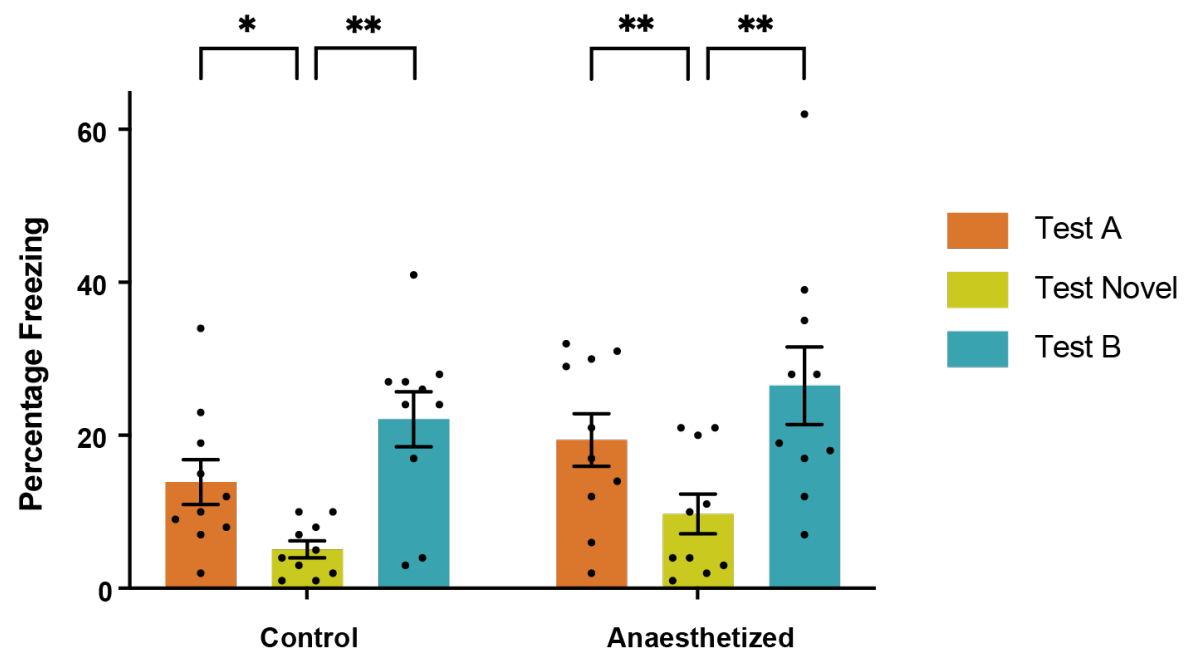

Figure S9. Memories of contexts can still be linked when context exposures are followed by anesthesia (as during structural imaging)

(a) Experimental setup: All mice were handled and habituated in a manner identical to the imaging experiments in figure 4. Mice experienced two contexts (A and B) 5 hours apart. Two hours following each context exposure, mice were anesthetized for 40 minutes to mimic anesthesia during the imaging sessions to study spine dynamics.

(b) The anesthetized mice can link the shock context (context B) to a neutral context (context A) 5 hours apart. For both groups of mice, freezing in the linked (context $A$ ), as well as training context (context $B$ ), is higher than freezing in a novel context. Therefore, prolonged anesthesia on the day of memory linking does not disrupt memory linking. (Two-way RM ANOVA, $F_{\text {context }}$ (1.5, $36)=27.8, P<0.0001$, Dunnett's multiple comparisons test).

Data represent mean \pm s.e.m. and each data point, ${ }^{*} p<0.05,{ }^{* *} p<0.01$. 


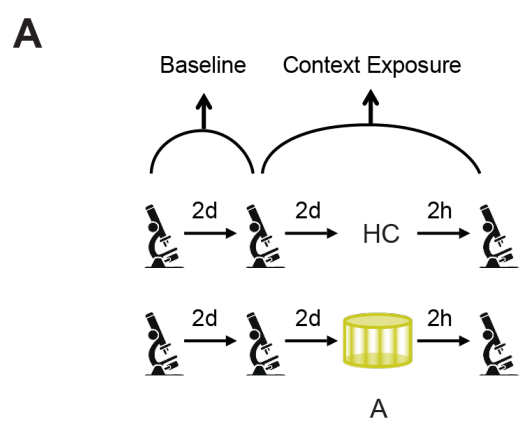

B
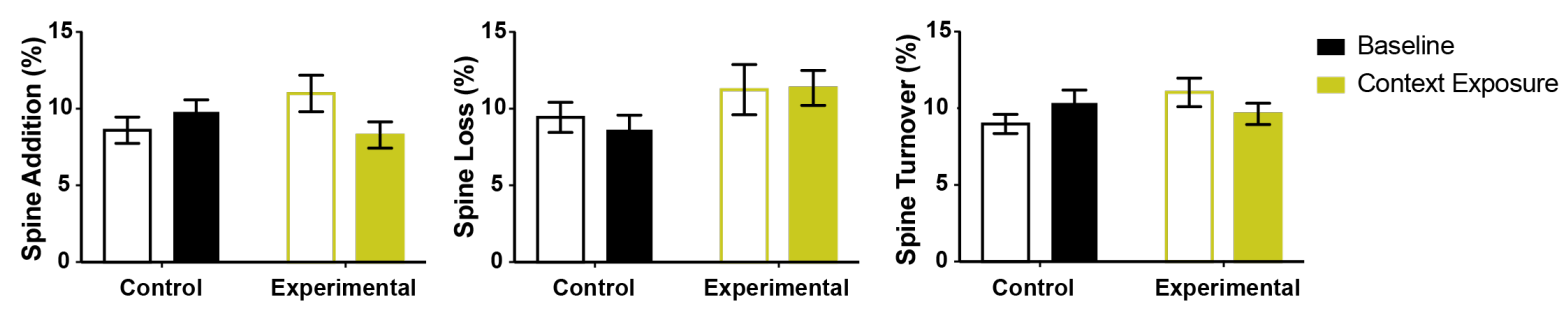

C
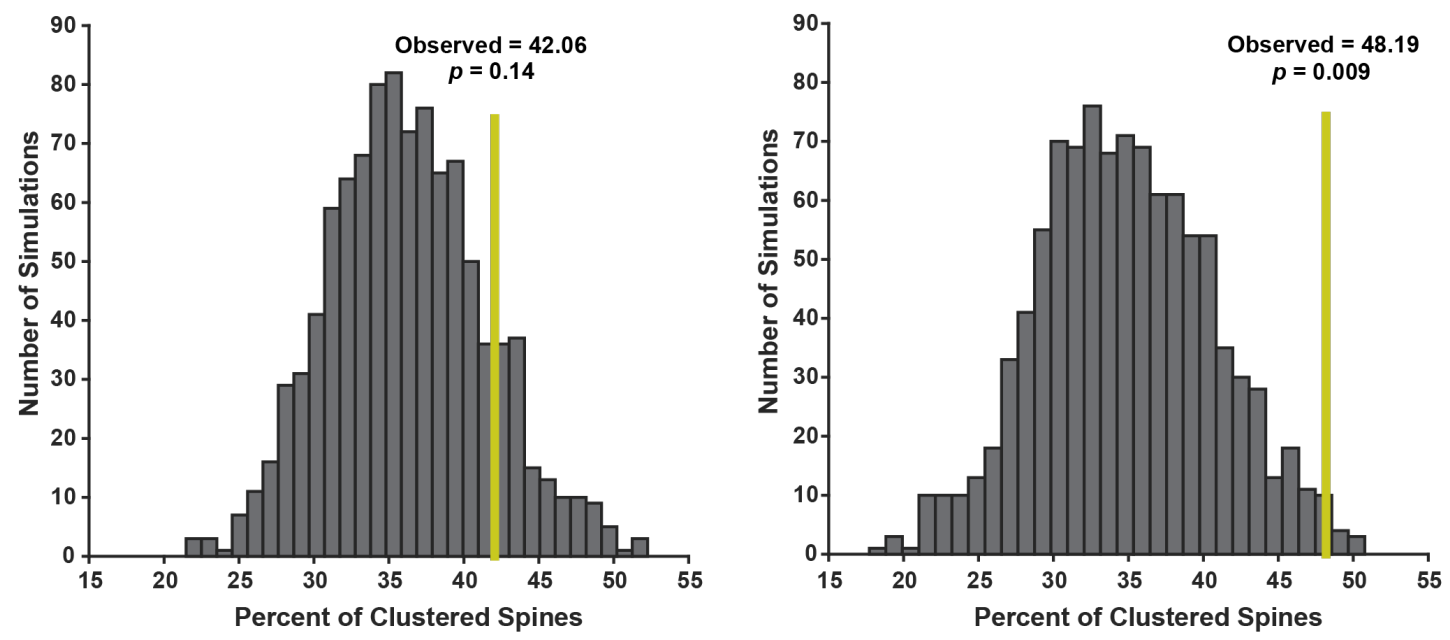

$$
\stackrel{2 \mathrm{~d}}{\longrightarrow} \stackrel{2 \mathrm{~d}}{\longrightarrow} \mathrm{HC} \stackrel{2 \mathrm{~h}}{\longrightarrow} \mathrm{l}_{\mathrm{i}}
$$

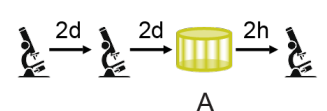

A

Figure S10. Spine dynamics within the RSC following context exposure

(a) Thy1-YFP mice were imaged every two days (baseline), and the same RSC dendrites were tracked to measure contextual exposure-related spine dynamics. Following two baseline imaging sessions, mice were left in the home cage or exposed to a novel context.

(b) Spine addition, spine loss, and spine turnover is not altered within the RSC apical dendrites following context exposure (Two-way RM ANOVA; Šídák's post hoc tests) 
(c) Clustered spine addition following context exposure is greater than chance: The histogram shows percent clustering from 1000 simulations of randomized new spine positions, where the percent of new spines within $5 \mu \mathrm{m}$ of each other was calculated. Yellow line: Percentage spine clustering observed from the data. The percentage of clustered spines is more than that expected by chance for the experimental group (Right, $\mathrm{n}=6 ; p=0.009$ ) whereas the percentage of clustered spines is at chance levels for the control group (Left, $n=5 ; p=0.17)$. Control: $n=44$ dendrites (5 mice); Experimental: $\mathrm{n}=46$ dendrites (6 mice).

Data represent mean \pm s.e.m. 
A

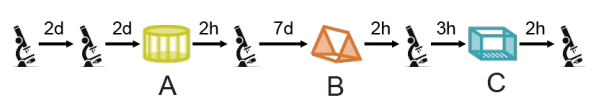

$$
\rho=0.04, p=0.8
$$
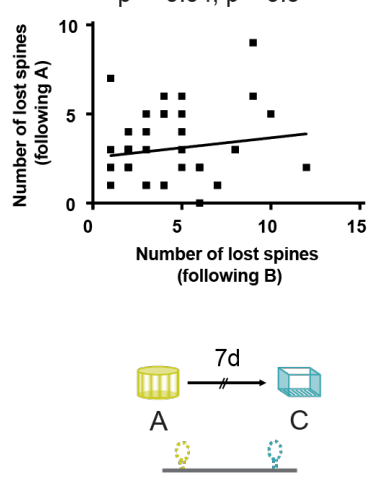

$\rho=0.09, p=0.5$
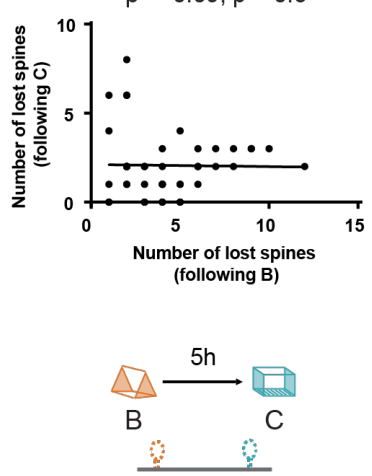

B

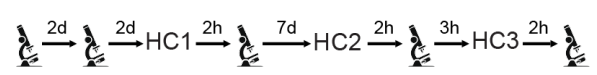
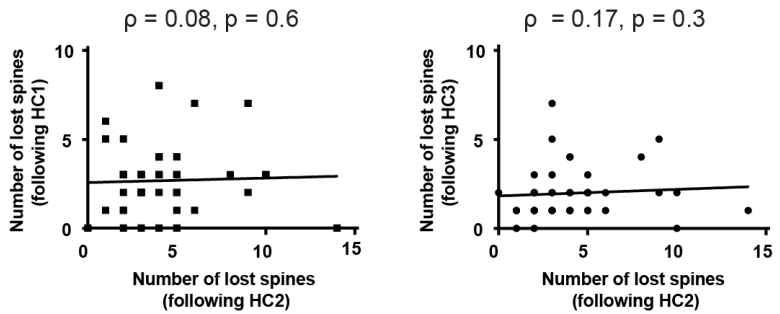

$$
\mathrm{HC} 1 \stackrel{7 \mathrm{~d}}{\longrightarrow} \mathrm{HC} 2
$$

$$
\mathrm{HC} 2 \stackrel{5 \mathrm{~h}}{\longrightarrow} \mathrm{HC} 3
$$

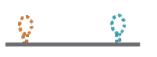

Figure S11. Spine loss during memory linking is not biased to the same dendritic segments

(a) Left: Number of spines lost from a dendritic segment following Context $A$ and $B$ exposure (7 days apart) are not correlated $(\rho=0.04, p=0.8)$. Right: Number of spines lost from a dendritic segment following Context B and C (5 hours apart) exposure are not correlated ( $\rho=0.09, p=$ $0.5)$.

(b) For mice left in their home cages $(\mathrm{HC})$, the number of spines lost from a dendritic segment are not correlated whether imaging sessions are separated by either 7 days (left, $\rho=0.08, p=0.6$ ) or 5 hours (right, $\rho=0.17, p=0.3$ ).

Spearman's correlation was used. 
A

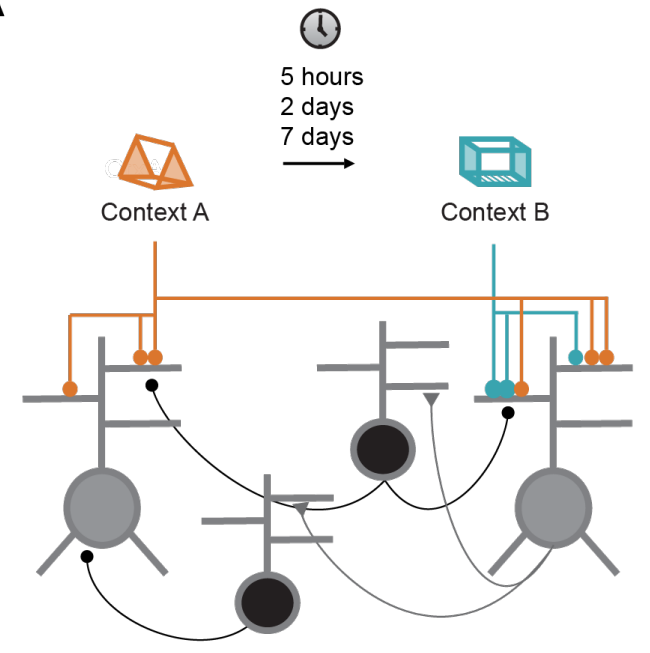

C

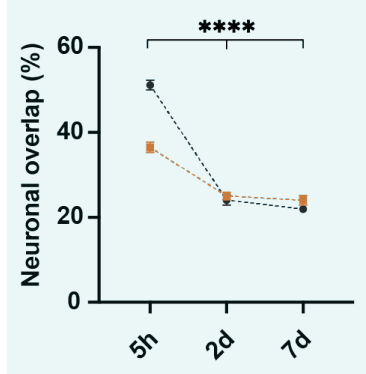

D

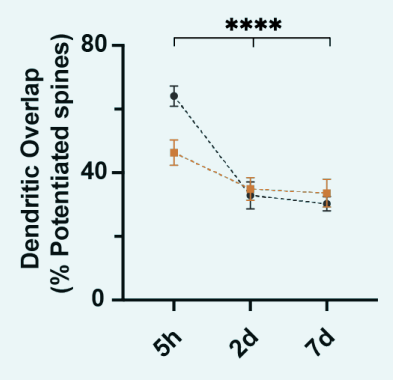

During Encoding
B

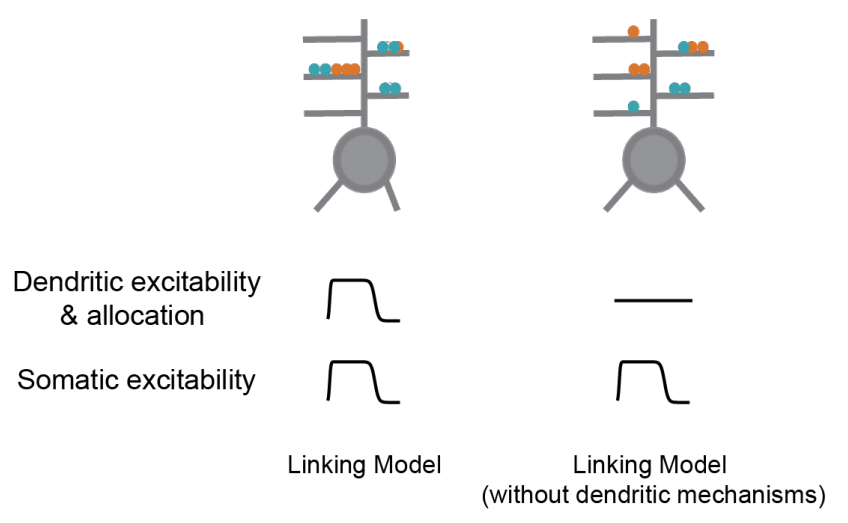

$\mathbf{E}$

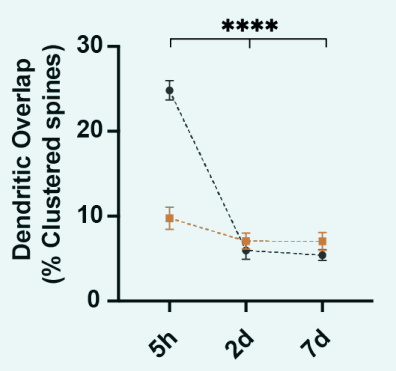

$\mathbf{F}$

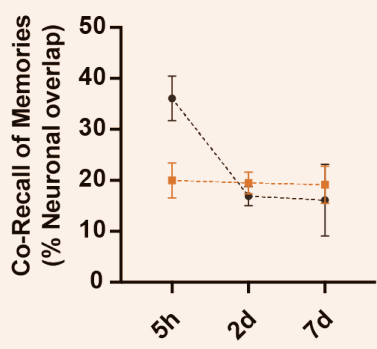

During Recall

- $\quad$ Linking Model

Linking Model (without dendritic mechanisms)

Figure S12. Dendritic mechanisms are necessary for linking memories acquired close in time in a spiking network model.

a) Schematic of the spiking network model. The network consists of 2-layer excitatory neurons (gray) with dendritic subunits, and subpopulations of dendrite-targeting and soma-targeting interneurons (black). See Methods for details.

b) Details of the learning-related plasticity mechanisms within the two network models: The linking model contains somatic and dendritic allocation mechanisms such that memory formation results in increases in somatic and dendritic excitability, and synapses are more likely to be potentiated in the presence of preexisting potentiated synapses on the same dendrite (see Methods). To assess the contribution of dendritic mechanisms to memory linking, learning-related changes in dendritic excitability and probability of synaptic potentiation are eliminated in the linking model without dendritic mechanisms.

c) Neuronal overlap during the encoding of two memories acquired 5 hours, 2 days or 7 days apart. When dendritic mechanisms (dendritic allocation mechanisms and increased dendritic 
excitability) are removed from the model, neuronal overlap during encoding is reduced when memories are acquired 5 hours (5h) apart.

d) Overlap between dendritic branches containing potentiated synapses following two memories acquired 5 hours, 2 days or 7 days apart. When dendritic mechanisms are removed from the model, overlap between dendritic branches with potentiated spines is reduced when memories are acquired 5 hours (5h) apart.

e) Overlap between dendritic branches containing newly added clustered spines following two memories acquired 5 hours, 2 days or 7 days apart. When dendritic mechanisms are removed from the model, overlap between dendritic branches with newly added clustered spines is reduced when memories are acquired 5 hours (5h) apart.

f) Co-recall of two memories as measured by neuronal overlap during recall. When dendritic mechanisms are removed from the model, co-recall of two memories (as measured by neuronal overlap during recall) is reduced when memories are acquired 5 hours (5h) apart. In addition, when dendritic mechanisms are removed from the model, neuronal overlap during recall is not different whether memories are acquired 5 hours, 2 days or 7 days apart indicating that dendritic mechanisms are necessary for linking of memories acquired close in time.

Two-way RM ANOVA. Dunnett's multiple comparisons for figure S12 c-e: linking model and linking model (without dendritic mechanisms): $5 \mathrm{~h}$ vs $2 \mathrm{~d}$ and $5 \mathrm{~h}$ vs $7 \mathrm{~d}$, all $p$ values $<0.0001$. For figure S12f, linking model: $5 \mathrm{~h}$ vs $2 \mathrm{~d}$ and $5 \mathrm{~h}$ vs $7 \mathrm{~d}$, all $p$ values $<0.0001$ but for linking model (without dendritic mechanisms): $5 \mathrm{~h}$ vs $2 \mathrm{~d}$ and $5 \mathrm{~h}$ vs $7 \mathrm{~d}$, all $p$ values $=$ non significant. Sidak's multiple comparisons for figure $6 \mathrm{~b}-\mathrm{e}$, overlaps at $5 \mathrm{~h}$ for linking model vs linking model (without dendritic mechanisms): all $p$ values $<0.0001$.

Data represent mean \pm s.e.m. of 10 simulation trials. For simplicity only comparisons within the linking model without dendritic mechanisms are presented (5h vs $2 \mathrm{~d}$ or $5 \mathrm{~h}$ vs $7 \mathrm{~d},{ }^{* * *} p<0.0001$ ). 


\section{Supplementary Table 1}

\begin{tabular}{|c|c|c|}
\hline \multicolumn{3}{|c|}{ Parameters of the computational model } \\
\hline$N_{\text {pyr }}$ & Number of excitatory neurons & 400 \\
\hline $\mathrm{N}_{\text {inh }}$ & Number of inhibitory neurons & $\begin{array}{l}50 \text { dendrite-targeting (DT) } \\
50 \text { soma-targeting (ST) }\end{array}$ \\
\hline $\mathrm{N}_{\text {dend }}$ & Number of dendritic subunits per neuron & $\begin{array}{r}10 \text { for excitatory } \\
1 \text { for interneurons }\end{array}$ \\
\hline $\mathrm{N}_{\mathrm{pyr} \rightarrow \mathrm{ST}}$ & $\begin{array}{l}\text { Synapses from excitatory neurons to soma- } \\
\text { targeting(ST) interneurons }\end{array}$ & $\begin{array}{l}\text { Count: } 1000 \\
\text { Weight: } 0.6\end{array}$ \\
\hline $\mathrm{N}_{\mathrm{pyr} \rightarrow \mathrm{DT}}$ & $\begin{array}{l}\text { Synapses from excitatory neurons to dendrite- } \\
\text { targeting (DT) }\end{array}$ & $\begin{array}{l}\text { Count: } 1000 \\
\text { Weight: } 0.3\end{array}$ \\
\hline $\mathrm{N}_{\mathrm{ST} \rightarrow \mathrm{pyr}}$ & Synapses from ST interneurons to excitatory neurons & $\begin{array}{l}\text { Count: } 10000 \\
\text { Weight: } 0.5\end{array}$ \\
\hline $\mathrm{N}_{\mathrm{DT} \rightarrow \mathrm{pyr}}$ & Synapses from DT interneurons to excitatory neurons & $\begin{array}{r}\text { Count: } 2000 \\
\text { Weight: } 0.3\end{array}$ \\
\hline $\mathrm{N}_{\text {input } \rightarrow \text { pyr }}$ & $\begin{array}{l}\text { Synapses from input afferents to pyramidal dendrites } \\
\text { per encoded memory }\end{array}$ & $\begin{array}{r}\text { Count: } 23000 \\
\text { Initial Weight: } 0.16-0.36\end{array}$ \\
\hline $\mathrm{E}_{\mathrm{L}}$ & Leakage reversal potential & $0 \mathrm{mV}$ \\
\hline$g_{\mathrm{E} / g_{I}}$ & Dendritic excitatory / inhibitory synaptic conductance & $22 n S / 20 n S$ \\
\hline$g_{L d} / g_{L}$ & Dendritic/somatic leak conductance & 10nS / 8nS \\
\hline$g_{\text {Inh }}$ & Somatic inhibitory current scaling constant & $600 n S$ \\
\hline $\mathrm{T}_{\text {/nh }}$ & Somatic inhibitory current time constant & $30 \mathrm{msec}$ \\
\hline$E_{E} / E_{l}$ & Excitatory /inhibitory synapse reversal potential & $+70 \mathrm{mV} /-10 \mathrm{mV}$ \\
\hline $\mathrm{C}$ & Membrane capacitance & $200 \mathrm{pF}$ \\
\hline$T_{\text {dend }}$ & Dendritic membrane time constant & $\begin{array}{l}\text { Inhibitory: } 20 \mathrm{msec} \\
\text { Excitatory: } 25 \mathrm{msec}\end{array}$ \\
\hline $\mathrm{V}_{\mathrm{d}}$ & Dendritic Depolarization & $-10 \mathrm{mV}<V_{d}<70 \mathrm{mV}$ \\
\hline$g_{\mathrm{ax}}$ & Axial conductance & $36 \mathrm{nS}$ \\
\hline$\theta_{\text {soma }}$ & Voltage threshold for somatic spikes & $18 \mathrm{mV}$ \\
\hline Tadapt & Adaptation time constant of excitatory neurons & $200 \mathrm{msec}$ \\
\hline$\beta_{\text {adapt }}$ & Adaptation reset constant & $\begin{array}{r}\text { Baseline excitability: } 9 \\
\text { High excitability: } 6.5\end{array}$ \\
\hline$a_{\text {adapt }}$ & Adaptation coupling parameter & 0.02 \\
\hline$\Theta_{\mathrm{PRP}}$ & $\begin{array}{l}\text { Calcium threshold for somatic Plasticity-Related } \\
\text { Protein (PRP) synthesis }\end{array}$ & 40.0 \\
\hline$\Theta_{\text {dend }}$ & Calcium threshold for dendritic excitability & 2.0 \\
\hline$\Theta_{\text {soma }}$ & Calcium threshold for somatic excitability & 40.0 \\
\hline $\mathrm{T}_{\mathrm{H}}$ & Time constant of homeostatic synaptic scaling & 1440 hours \\
\hline
\end{tabular}




\section{Methods}

Animals. All experimental protocols were approved by the Chancellor's Animal Research Committee of the University of California, Los Angeles, in accordance with NIH guidelines. cFostTa mice that express tetracycline transactivator (tTA) protein under the control of the c-fos (also known as Fos) promoter were maintained in a C57BL/6N background. Adult (3-8 months old) male and female Thy1-YFP-H mice (Jackson Laboratories, Stock No: 003782) were used for structural imaging experiments. C57BL/6N Tac mice were purchased from Taconic Farms (Germantown, NY) for all other experiments.

Viral construct. pAAV.Syn.GCaMP6f.WPRE.SV40 was a gift from Douglas Kim \& GENIE Project (Addgene viral prep \# 100837-AAV1; http://n2t.net/addgene:100837; RRID: Addgene 100837). The lentivirus hM3Dq.T2A.EGFP vector was derived as previously described in Cai et al., 2016 (4). Finally, AAV1.TRE.hChR2.mCherry, AAV1.TRE.hChR2.mCherry.DTE and AAV1.TRE.mCherry.DTE were derived in our laboratory. Briefly, to construct a vector for TREdriven hChR2 expression, a CamKIla promoter from pAAV-CamKIla-hChR2(H143R)-mCherry (Addgene \#26975) was replaced with TRE promoter from pAAV-RAM-d2tTA::TRE-NLS-mKate2WPRE (Addgene \#84474) using Mlul/Agel digestion. The DTE sequence of Arc mRNA was PCRamplified from cDNA of 14 weeks Spraque-Dawley rat using primers as previously described (8) and inserted into the pAAV-TRE-hChR2-mCherry vector using EcoRI/HindIII. The pAAV-TREhChR2-mCherry and pAAV-TRE-hChR2-mCherry-DTE were subjected to Agel/BsrGI digestion for construction of mCherry vectors, respectively. The mCherry was digested by Agel/BsrGI from pmCherry-N1. AAV production was conducted as previously described in detail (37) with modifications.

Surgery. Mice were anesthetized with 1.5 to $2.0 \%$ isoflurane for surgical procedures and placed into a stereotactic frame (David Kopf Instruments, Tujunga, CA) on a heating pad. Artificial tears were applied to the eyes to prevent drying. Subcutaneous saline injections were administered throughout each surgical procedure to prevent dehydration. In addition, carprofen 
(5 mg kg-1) and dexamethasone $(0.2 \mathrm{mg} \mathrm{kg}-1)$ were administered both during surgery and for 2-7 days post-surgery. A midline incision was made down the scalp, and a craniotomy was performed with a dental drill. Water with amoxicillin was administered for two weeks.

Miniscope experiments. Surgeries. Mice were unilaterally microinjected with $500 \mathrm{nl}$ of AAV1.Syn.GCaMP6f.WPRE.SV40 virus at 20-120 $\mathrm{nl} / \mathrm{min}$ into the $\mathrm{RSC}$ using the stereotactic coordinates: $-2.3 \mathrm{~mm}$ posterior to bregma, $0.5 \mathrm{~mm}$ lateral to the midline, and $-0.8 \mathrm{~mm}$ ventral to the skull surface. Immediately afterward, the microendoscope (a gradient refractive index or GRIN lens) was implanted above the injection site. For this procedure, a $2.0 \mathrm{~mm}$ diameter circular craniotomy was centered above the virus injection site. The microendoscope $(0.25$ pitch, 0.50 $\mathrm{NA}, 2.0 \mathrm{~mm}$ in diameter, and $4.79 \mathrm{~mm}$ in length, Grintech $\mathrm{Gmbh}$ ) was slowly lowered with a stereotaxic arm above the craniotomy $450 \mu \mathrm{m}$ ventral to the surface of the skull. Next, a skull screw was used to anchor the microendoscope to the skull. Both the microendoscope and skull screw were fixed with cyanoacrylate and dental cement. Kwik-Sil (World Precision Instruments) was used to cover the microendoscope. Three weeks later, a small aluminum baseplate was cemented onto the animal's head atop the previously placed dental cement. The microscope was placed on top of the baseplate and locked in a position such that the field of view contained cells and visible landmarks, such as blood vessels, and these appeared sharp and in focus. Finally, a plastic headcap was fit into the baseplate and secured with magnets. Independent experiments confirmed that GCaMP6f expression was limited to RSC neurons (figure 1b).

Miniscope Behavior. Using head-mounted miniature microscopes (UCLA Miniscopes V3)(4, 38), we imaged GCaMP6f-mediated calcium dynamics in RSC neurons of GRIN lens implanted mice that explored distinct contexts. Before imaging sessions, mice were handled and habituated to the experimental conditions, including carrying the Miniscope while it was tethered to the implanted GRIN lens. Mice were exposed to each context (with distinct visual, auditory, and olfactory cues) for 10 mins during which calcium transients were recorded (figure 1c for 
representative calcium transients). Context A was separated from Context B by 7 days, and Context $B$ and Context $C$ were separated by 5 hours (figure 1e).

Miniscope Analysis. Calcium imaging data were registered to remove small movement artifacts using NormCorre (39). This was followed by automated segmentation, demixing, and denoising of calcium signals using constrained non-negative matrix factorization for endoscopic data (CNMFe) (40). We used a modified version of the Miniscope analysis package developed by Guillaume Etter (Sylvain Williams Lab, McGill University) for data analysis (41). Recordings from multiple sessions of the same mouse were aligned using an amplitude-based registration algorithm used for within-session registration, except the algorithm was only applied to the mean frame from each session. Once regions of interest (ROls- putative neurons) from two sessions were registered, ROls across two sessions were matched to each other using a distance (between ROI centroids) and correlation (between ROls spatial footprints - SFPs) measure. The neuronal ensemble overlap was calculated as the percentage of ROls activated in both contexts divided by the average number of ROls identified in each imaging session. Neurons were matched across days based on distance (< 4 pixels) and correlation $(>0.9)$ thresholds. These results (figure 1d) were consistent and robust for a range of distance (3-9 pixels) and correlation (0.6-0.95) thresholds used to match segments across days (data not shown).

In a parallel approach, we aligned and concatenated the imaging data from the three context exposures into a single video file (followed by motion correction and segmentation as described above) and analyzed the data such that we were able to detect and track the activity of ROls across all different sessions as well as investigate their modulation during context exploration. The raw data from CNMFe extracted putative neurons was deconvolved into spike probabilities using the foopsi thresholded method (OASIS toolbox) (42). Finally, the spike probabilities from single frames were binarized between 1 (active) and 0 (inactive). For each neuron, the firing rate (number of active frames per second) for each session was calculated. 
Population Vector Correlations (PVCs) were calculated as the Pearson correlation between the average firing rate (per session) of each neuron across two imaging sessions (figure S2).

Naïve Bayes (NB) Binary Classifier. The activity of each neuron during each 10 min session was resampled into various time bin sizes (0.5-60 seconds bins, step size $0.5 \mathrm{~s})$. Each resampled data with a specific bin size was used as trials from each session, so the NB classifier would be able to use the information contained in the probability of activity from each neuron between two given sessions to sort between them. The quality of the classification is defined by the area under the receiver operating characteristic curve (AUC), which ranges from 0 to $1 . A \cup C$ $=\sim 0.5$ means sorting at chance levels by the classifier.

Pairwise correlations (PWC) maps for each session were calculated by binning neuronal activity into $100 \mathrm{~ms}$ bins to compute the Pearson correlation for each pair of neurons (figure S4a,b). PWC stability was calculated as the Pearson correlation between PWC maps from different sessions, excluding the main diagonal (correlation between each neuron with itself) (22). Since artificially high correlations can arise due to sub-optimal demixing of calcium signals from nearby ROIs, we computed the PWC analysis while ignoring the PWCs from nearby cell pairs. We defined nearby cells as cell pairs where spatial footprints (SFP) had any overlap or where the centroid to centroid distance was shorter or equal to 20 pixels $(\sim 40 \mu \mathrm{m})$. To control for the different number of neurons detected for different mice, we calculated PWC stability between 2 sessions by randomly subsampling a group of 10 cells, computing the PWC map for each of the sessions using these cells, and computing the Pearson correlation between the two PWC maps. This process was repeated 1000 times and the final PWC stability was defined as the average of these 1000 values. The absolute PWC per imaging session and PWC stability across sessions follows the same trend whether the analyses were done with or without nearby cells. For brevity, we only present analyses that excluded the nearby neurons.

Optogenetic experiments. Adult male and female (3-8 months) cFos-tTa transgenic and their wild-type littermates maintained on doxycycline chow (for 1 month or more) were bilaterally 
microinjected with $500 \mathrm{nl}$ of AAV1.TRE.hChR2.mCherry or AAV1.TRE.hChR2.mCherry.DTE or AAV1.TRE.mCherry.DTE virus at $20-50 \mathrm{nl} \mathrm{min}^{-1}$ into the RSC using the stereotactic coordinates: $-2.3 \mathrm{~mm}$ posterior to bregma, $0.5 \mathrm{~mm}$ lateral to the midline, and $-0.8 \mathrm{~mm}$ ventral to the skull surface. Following viral injections, bilateral optogenetic cannulae (Doric Lenses Inc.; DFC_200/240-0.22_0.5mm_GS1.0_FLT) were implanted over the injection site at $-0.45 \mathrm{~mm}$ ventral to the skull surface. Mice were allowed to recover from surgeries for 3-5 weeks before being handled ( 3 days) and habituated (3-5 days) for behavioral exposure as well as optogenetic manipulation. The day after the last day of habituation, mice were taken off doxycycline chow and placed on regular chow and tTA expression was allowed for 3 days before behavioral tagging for the memory linking experiments. The activity-dependent tag was shut off by administration of high dox chow $\left(1 \mathrm{~g} \mathrm{~kg}^{-1}\right) 90$ minutes after behavioral tagging.

Chemogenetic experiments. Adult (3-5 months old) C57BI/6NTac male mice were bilaterally microinjected with $1000 \mathrm{nl}$ of lentivirus hM3Dq.T2A.EGFP at 20-100 $\mathrm{nl} / \mathrm{min}$ into the RSC using the stereotactic coordinates: -1.95 and $-2.65 \mathrm{~mm}$ posterior to bregma, $0.5 \mathrm{~mm}$ lateral to the midline, and $-0.8 \mathrm{~mm}$ ventral to the skull surface. Following viral injections, mice were allowed to recover from surgeries for 3 weeks before being handled ( 3 days) and habituated ( 3 days) for a modified two-day memory linking experiment. To ensure that the same RSC neurons are recruited for encoding these different contexts, we transiently increased the intrinsic excitability of a small subset of RSC neurons by administering a clozapine $\mathrm{N}$-oxide $(\mathrm{CNO}, 0.5 \mathrm{mg} / \mathrm{kg})$ injection 45 mins before each context exploration (4). The control mice only received the CNO injection before the second context exploration. Following this, the mice underwent the memory linking paradigm described below.

Memory linking studies. Linking of context memories was carried out as previously described (4). For experiments investigating the effect of manipulating neuronal (figure 2c) or dendritic overlap (figure 5) on memory linking, the two context exposures were separated by 2 days. We did this for two reasons. First, two contexts explored 1 day apart are linked but when 
contexts are explored 2 days apart, they are not linked (4). Second, Channelrhodopsin expression under the TetTag system peaks at 24 hours (43). This allowed us to extend the window of memory linking (to 2 days) by using the transient expression of Channelrhodopsin on a day after the first context exposure.

Briefly, mice were handled for 3 days (2-5 min/day) and then habituated to transportation and experimental room/s for 3-5 days (2-5 mins/ day). In the memory linking task, mice explored 2 distinct contexts ( $A$ and then $B$, for 10 mins each) separated by 5 h (figure $2 b, S 6$ ) or 2 days (figure 2c, figure 5, figure S7). For figure 2b: The context exposure in chamber B also included a 2s, $0.75 \mathrm{~mA}$ footshock that was delivered 58 seconds before the end of context exposure. For figures 2c and 5: Two days following the last context exposure (in B), mice were placed in context B again for an immediate foot shock (10 second baseline, 2 second shock, 0.7-0.75mA, 28-58 second post-shock period). For figure S7: To compensate for the lower freezing seen in C57BI/6N Jackson mice (the genetic background of the Thy1-YFP mice), the immediate shock protocol was modified to a 10 second baseline, two shocks for 2 seconds each, $0.75 \mathrm{~mA}, 15$ seconds apart. For the context tests, mice were returned to the designated contexts for 5 minutes each. Freezing was assessed via an automated scoring system (Med Associates) with 30 frames per second sampling rate; the mice needed to freeze continuously for at least one second before freezing could be counted.

Optogenetic testing. Mice were placed in an open field and freezing behavior was recorded using a digital camera. Following a 3-minute baseline period, the tagged RSC ensemble was reactivated using a $473 \mathrm{~nm}$ laser $(5 \mathrm{~ms}$ pulses, $5 \mathrm{~Hz}$ ) for one minute followed by a one minute interval with no stimulation. This pattern of stimulation was repeated three times, and the time spent freezing during the three epochs was averaged.

Structural $2 p$ imaging. Methods. Adult (3-8 months old) male and female Thy1-YFP-H mice were used for structural imaging experiments. Mice underwent window implantation surgeries as previously described (29). Briefly, a square region of the skull 2-3 $\mathrm{mm}$ in width was 
marked using stereotactic coordinates (RSC: center at bregma $-2.3 \mathrm{~mm} \mathrm{AP}$ ). The skull was thinned using a dental drill and removed. After cleaning the surgical site with saline, a custom cut sterilized coverslip (square, $2 \times 2 \mathrm{~mm}$ unilateral or $3 \times 3 \mathrm{~mm}$ bilateral) was placed on the dural surface and fastened with adhesive and dental acrylics to expose a square window of approximately 2 $\mathrm{mm}$. Next, an aluminum bar with a threaded hole was attached to stabilize the mice during imaging sessions. Mice were allowed to recover for two or more weeks before the first imaging session. Following recovery from surgery, mice were handled and habituated as per the memory linking paradigm. In addition, mice were also habituated for transportation to the imaging room as well as anesthesia. After handling/habituation (1-2 days later), mice underwent the first home cage baseline imaging session. Two days later mice underwent the second baseline imaging session. Two days following the last baseline imaging session, a subset of mice were exposed to a novel context 'A' for 10 minutes while another subgroup remained in the home cage (control group). After 7 days, mice were exposed to a novel context (B, 10 minutes) which was followed by a third novel context exposure (C, 10 minutes) five hours later. Mice were imaged 2 hours after each context exposure.

Two-Photon imaging measuring spine dynamics. A custom-built two-photon laser scanning microscope was paired with a Spectra-Physics two-photon laser tuned to $920 \mathrm{~nm}$. A 40x 1.0 NA water immersion objective (Zeiss) was used to acquire images 2 hours after each behavioral session. Mice were lightly anesthetized with isoflurane and attached to the head mount using a small screw. During the first imaging session, segments of apical dendrites from Layer $\mathrm{V}$ pyramidal cells were imaged. These segments were acquired within $200 \mu \mathrm{m}$ from the cortical surface, likely representing dendrites located in layers I and II/III. Imaged segments were generally oriented in the $x, y$ plane of imaging with minimal z-projection. 512x512 pixel images were acquired at $0.5 \mu \mathrm{m}$ intervals to fully capture the segment of dendrite, and image stacks generally consisting of 30-40 slices. If a segment of dendrite was larger than could be acquired 
in one 512x512 pixel stack, additional image stacks were sequentially acquired through the $x, y, z$ plane of the dendrite in question so that its full extent could be visualized. The same segments were repeatedly imaged across experimental days by locating their position via a coordinate system established during the first imaging session.

Image and Data Analysis. Dendritic spines were analyzed and counted by established criteria. Specifically, the Spine Analysis software included in Scanlmage was used to open all imaging days for a given segment of dendrite. A segment is classified as the entire visible length of a piece of a dendrite, and segments were often followed across several images. The presence, gain, and loss of spines was quantified across days for each segment, and all segments were examined for a given animal. Importantly, all images were coded following the completion of the experiment so that the experimenter was blind to the training status of all mice while analyzing and counting spines.

Clustering ratios were calculated as the number of clustered spines divided by the total number of new spines gained between two time points. Clustered spines were defined as a new spine that was less than $5 \mu \mathrm{m}$ from another new spine. The data did not follow a normal distribution, and therefore correlations were calculated using Spearman's rho ( $\rho)$. For the resampling analysis of clustering, the number of new spines added per segment of dendrite was used to pick an equivalent number of random positions along the same segment and assess whether these positions were within $5 \mu \mathrm{m}$ of each other. When this was completed for all dendrites for a given animal, the percent of clustered spines was calculated as the number of randomly selected new spine positions within $5 \mu \mathrm{m}$ of each other divided by the total number of stably added new spines for that animal. In turn, each animal's resampled clustering percentage was calculated, and then these values were averaged together. This completed one resampling event, and this process was then repeated for a total of 1000 resampling events, which then yieded the full distribution of random sampling (figure S10).

Cross Clustering across exposures. The number of clustered spines added following a 
context exposure were randomly distributed on the dendritic segments from that mouse $(10,000 x)$.

The percentage of clustered spines added to a dendritic segment following the first context exposure, that were added to a segment that also gained clustered spines following the subsequent context exposure, were measured and compared to the shuffled distribution obtained from the above analysis.

Functional two-photon Imaging. Mice underwent bilateral injection of 1:10 dilution of GCaMP6f in the RSC (as described in figure1). A square $3 \mathrm{~mm} \times 3 \mathrm{~mm}$ craniotomy spanning the midline and hence revealing both RSCs was then made over the injection. After cleaning the surgical site with saline, the coverslip was placed on the dural surface and fastened with adhesive and dental acrylics to expose a square window. Next, an aluminum bar with a threaded hole was attached to stabilize the mice during imaging sessions. Two to three weeks following the surgery, mice underwent handling ( 3 days) and habituation ( 3 days) to acclimate to the treadmill and headfixation. Neuronal and dendritic calcium activity was imaged in head-fixed mice that were free to run on a head-fixed setup.

We recorded dendritic signals during context exposure as well as those evoked spontaneously using a resonant-scanning two-photon microscope (Neurolabware) controlled by Scanbox acquisition software. Distinct contexts were created by immobilizing the mice either on a running wheel, a treadmill, or a horizontal disc (figure S8), in addition to distinct auditory, olfactory and visual cues associated with each context. Visual stimuli were presented on a large LCD monitor directly in front of the animal and $18 \mathrm{~cm}$ from the eye. Visual stimuli consisted of non-repeating natural movies with intermittent gray screens (9s on, 14s off). Spontaneous response data was collected with a blank gray screen in the absence of auditory and olfactory cues. A Coherent Discovery laser (Coherent Inc.) was used for GCaMP excitation, fixed at a wavelength of $920 \mathrm{~nm}$. The objective used was a 16x water-immersion lens (Nikon, 0.8NA, $3 \mathrm{~mm}$ working distance). Image sequences were captured at $15.5 \mathrm{~Hz}$ at a depth of $<100 \mu \mathrm{m}$ below the pia. 
Collected data were processed using the Suite2P analysis pipeline (44). Recorded frames were aligned using a non-rigid motion correction algorithm. Following alignment, any frames with significant motion in the z-axis were dropped from the original video and the data were reanalyzed. Regions of interest (representing dendritic segments) were segmented in a semi-automated manner using a Suite $2 p$ based classifier. Dendritic segments were matched across imaging sessions using an open-source algorithm (https://github.com/ransona/ROIMatchPub, matching criteria: correlation: 0.4 ). The percentage of reactivated dendrites was defined as the number of matched segments normalized to the average number of dendritic segments detected in each imaging session.

Computational modeling. We adapted a previously published model network of memory allocation (13). The model network consists of populations of excitatory and inhibitory neurons, which are modeled as 2-level integrators to account for dendrites. Neurons consist of a somatic unit connected to multiple independent dendritic subunits. Both dendrites and soma are modeled using simplified integrate-and-fire model dynamics, where the somatic unit includes adaptation current, and dendrites and soma are coupled via axial resistance. Inhibitory neurons provide feedback inhibition and are separated into 2 equal sub-populations, soma-targeting and dendritetargeting. The voltage of each dendritic subunit is as follows:

$C \frac{d V_{d}}{d t}=-g_{L}\left(V_{d}-E_{L}\right)+a_{e x c} g_{E} u_{E}(t)\left(E_{E}-V_{d}\right)-g_{I} u_{I}(t)\left(E_{I}-V_{d}\right)$

where $V_{d}$ is the dendritic depolarization, $C$ is the membrane capacitance, $E_{E}$ is the reversal potential for excitatory receptors, $E_{l}$ is the reversal potential for inhibitory receptors, $E_{L}$ is the resting potential $(0 \mathrm{mV}), a_{e x c}$ is the dendritic excitability level parameter, $g_{L}$ is the leak conductance, $g_{E}, g_{l}$ are the maximal excitatory and inhibitory synaptic conductances. $u_{l}(t) / u_{E}(t)$ are the instantaneous activations of excitatory and inhibitory synapses on the dendrite respectively: $u_{E / I}(t)=\sum_{j} w_{j} \delta\left(t-t_{j}\right)$

where $w_{j}$ is the weight of synapse $j$ and $t_{j}$ are the timings of incoming spikes. 
Somatic voltage is modeled as an Integrate-and-Fire model with adaptation as follows:

$$
\begin{aligned}
& C \frac{d V}{d t}=-g_{L}\left(V-E_{L}\right)+I_{\text {noise }}(t)+I_{\text {ax }}(t)-I_{\text {inh }}(t)-I_{\text {adapt }}(t) \\
& \tau_{\text {adapt }} \frac{d I_{\text {adapt }}}{d t}=\alpha_{\text {adapt }}\left(V-E_{L}\right)+\beta_{\text {adapt }} \delta\left(t-t_{\text {spike }}\right)-I_{\text {adapt }} \\
& I_{\text {ax }}=\sum_{i} g_{a x}\left(V_{d, i}-V\right)_{+} \\
& \tau_{\text {inh }} \frac{d I_{\text {inh }}}{d t}=\sum_{i} g_{\text {inh }} \delta\left(t-t_{i}\right)-I_{\text {inh }}
\end{aligned}
$$

where $V$ is the somatic voltage, $I_{\text {noise }}$ is uniform noise current (max amplitude $500 \mathrm{pA}$ ), $l_{a x}$ is the excitatory axial current, $l_{\text {inh }}$ is the filtered inhibitory current from somatically-targeting interneurons, $l_{\text {adapt }}$ is the adaptation current, $T_{\text {adapt }}$ is the adaptation time constant, $\alpha_{\text {adapt }}$ the adaptation coupling parameter, $\beta_{\text {adapt }}$ is the amount by which adaptation current increases every time the neuron spikes, $g_{a x}$ is the axial resistance, $\tau_{i n h}$ is the time constant of inhibitory current and $g_{i n h}$ the inhibitory current scaling constant. Somatic spiking occurs when the somatic voltage reaches the spike threshold $\theta_{\text {soma. }}$. On an incoming spike, synaptic and dendritic branch calcium is increased by an amount $\Delta C \alpha\left(V_{d}\right)$ that depends on the instantaneous depolarization $V_{d}$ of the dendritic branch to account for the Magnesium blocking of NMDA receptor:

$\Delta C a\left(V_{d}\right)=\frac{1}{1+\frac{e^{-0.07\left(V_{d^{-}} 70\right)}}{9}}$

Synaptic inputs representing memories to be encoded are initially allocated randomly to the dendritic subunits of excitatory neurons with initial weight ranging uniformly randomly between 0.16 and 0.36 . In addition, feedback synapses between excitatory and inhibitory populations are allocated at random, with separate distributions for soma-targeting and dendrite-targeting interneurons (see Supplementary Table 1).

The total calcium influx during memory encoding determines synaptic plasticity, changes in excitability and the levels of plasticity-related proteins (PRPs) after encoding. Synapses that receive calcium influx above zero during stimulation are selected for potentiation or depression. 
If the synapse resides on a neuron that is highly activated (spiking with frequency $>10 \mathrm{~Hz}$ during stimulus presentation) then the synapse is tagged for potentiation with probability $p_{L T P}=0.29+$ $X_{\text {dend }}{ }^{*} N_{s} / 2$, otherwise the synapse is tagged for depression. $N_{s}$ is the number of preexisting potentiated synapses in the same dendrite and $X_{\text {dend }}$ is the excitability of the dendrite (see below). Synaptic tags decay exponentially with time constant of 1 hour.

When the somatic calcium level of a neuron exceeds $\Theta_{P R P}$ at time $T$, the level of plasticity-related proteins of the neuron is elevated according to the following function:

$\operatorname{PRP}(T)=\left\{\begin{array}{rr}\frac{T-20}{30} e^{-\frac{T+10}{30},} & T>20 \\ 0, & T \leq 20\end{array}\right.$

where $T$ is the time since the stimulus in minutes. When synaptic tags and PRPs have values above 0.1 , the weights $w$ of synapses are updated by $\Delta w=0.15^{\star} P R P(t)^{\star}($ synaptic tag), where $\mathrm{t}$ is time in seconds. Synaptic weights are bounded in the interval $[0,1]$. We note that, because the delay between memories in all simulations is 5 hours or more, the PRP protein levels are always sufficient for full synaptic tag consolidation, and thus there was no competition for PRPs that could affect the linking of memories.

Excitability within the Linking model: When the total dendritic and somatic calcium are above thresholds $\Theta_{\text {dend }}$ and $\Theta_{\text {soma }}$ respectively, the excitability level $X$ of the dendrite/soma is increased by the amount given by:

$X_{\text {dend } / \operatorname{soma}}(T)=\frac{1}{1+e^{-3(T-1)}}-\frac{1}{1+e^{-(T-26)}}$

where $T$ is the time in hours since the stimulus. The excitability level parameter $a_{\text {exc }}$ is increased by $10 \%$ when $X_{\text {dend }}>0.1$, while the adaptation reset parameter $\beta_{\text {adapt }}$ is increased by $28 \%$ when $X_{\text {soma }}>0.1$. For the simulations of the Linking model without dendritic mechanisms, the dendritic excitability was set at $X_{d e n d}=0$ and the probability $p_{L T P}$ was kept constant at 0.32 .

Synaptic weights are additionally subject to a homeostatic synaptic scaling rule, which adjusts synaptic weights $w_{j}$ to normalize the total synaptic input to each neuron with time constant $\tau_{H}$ : 
$\frac{d w_{j}}{d t}=\frac{1}{\tau_{H}}\left(1-\frac{\sum_{j} w_{j}}{w_{\text {init }} N_{\text {syn }}}\right)$

where $w_{\text {init }}$ is 0.3 and $N_{\text {syn }}$ is the total number of incoming synapses to the neuron.

Stimulation protocol: For every memory being encoded, the synaptic inputs which represent the memory are stimulated for 4 seconds with firing rate $35 \mathrm{~Hz}$ in order to drive the initially weak synapses. After the first memory encoding, a delay period is simulated for 5 hours, 2 days or 7 days, and then the second memory is encoded. Memories are recalled by being stimulated again after 2 days.

For the clustering statistics, a branch was considered to contain overlapping clusters if it contained at least 3 potentiated synapses from each memory. The parameters used in the model are listed in Supplementary Table 1. The model was written in $\mathrm{C}++$ and data analysis was done with Python/numpy. The source code for the simulation, data analysis and scripts to reproduce the data and figures are available at https://dendrites.gr/wp-content/uploads/2021/11/rsc model.zip.

Quantification and Statistical Analyses. The investigator who collected and analyzed the data including behavior, imaging and staining were blinded to the mouse genotypes and treatment conditions. Error bars in the figures indicate the SEM. All statistical analyses were performed using GraphPad Prism 9. For behavior and imaging experiments, $\mathrm{n}$ designates the number of mice unless otherwise mentioned. Statistical significance was assessed by Student's t test, or one- or two-way ANOVA where appropriate, followed by the indicated post-hoc tests. The level of significance was set at $p<0.05$.

Acknowledgments. We thank P. Riazi, J. Lin, E. Rubin, G. Padda, Y. Cai, M. Zhou, D. J. Cai, D. Aharoni, C.E. Yaeger, G. Fernandes, A. F. de Sousa, A. Chowdhury and Y. Shen for advice and technical support. We thank Dean Buonomono, Mayank R. Mehta, Peyman Golshani and James M. Otis for helpful discussions. This work was supported by grants from the NIMH (R01 MH113071), NIA (R01 AG013622), and from the Dr. Miriam and Sheldon G. Adelson Medical Research Foundation to AJS. The computational modelling work was supported by the 
European Commission (H2020-FETOPEN-2018-2019-2020-01, FET-Open Challenging Current Thinking, GA-863245), the NIH (R01MH124867-01) and the Einstein Foundation Berlin.

Author contribution. MS and AJS did experimental design, data acquisition and analyses, drafting and revising the article; JT helped with experimental design for two-photon calcium imaging experiments. SM, IDM, AP helped with behavioral data acquisition; SK, JL, WDH made the TRE-dependent constructs; DAF helped with Miniscope data analysis and interpretation; $\mathrm{AL}$ and $\mathrm{SH}$ helped with two-photon data analyses and interpretation; GK and PP designed, implemented, and analyzed the computational modelling experiments. All authors read and edited the manuscript.

Competing interests. The authors declare no competing interests. 
1. T. Rogerson, D. J. Cai, A. Frank, Y. Sano, J. Shobe, M. F. Lopez-Aranda, A. J. Silva, Synaptic tagging during memory allocation. Nat Rev Neurosci. 15, 157-169 (2014).

2. M. Sehgal, M. Zhou, A. Lavi, S. Huang, Y. Zhou, A. J. Silva, Memory allocation mechanisms underlie memory linking across time. Neurobiology of Learning and Memory. 153, 21-25 (2018).

3. A. F. de Sousa, A. Chowdhury, A. J. Silva, Dimensions and mechanisms of memory organization. Neuron, S0896-6273(21)00455-4 (2021).

4. D. J. Cai, D. Aharoni, T. Shuman, J. Shobe, J. Biane, W. Song, B. Wei, M. Veshkini, M. LaVu, J. Lou, S. Flores, I. Kim, Y. Sano, M. Zhou, K. Baumgaertel, A. Lavi, M. Kamata, M. Tuszynski, M. Mayford, P. Golshani, A. J. Silva, A shared neural ensemble links distinct contextual memories encoded close in time. Nature. 534, 115-118 (2016).

5. A. J. Rashid, C. Yan, V. Mercaldo, H.-L. (Liz) Hsiang, S. Park, C. J. Cole, A. D. Cristofaro, J. Yu, C. Ramakrishnan, S. Y. Lee, K. Deisseroth, P. W. Frankland, S. A. Josselyn, Competition between engrams influences fear memory formation and recall. Science. 353, 383-387 (2016).

6. J. Yokose, R. Okubo-Suzuki, M. Nomoto, N. Ohkawa, H. Nishizono, A. Suzuki, M. Matsuo, S. Tsujimura, Y. Takahashi, M. Nagase, A. M. Watabe, M. Sasahara, F. Kato, K. Inokuchi, Overlapping memory trace indispensable for linking, but not recalling, individual memories. Science. 355, 398-403 (2017).

7. T. J. Ryan, D. S. Roy, M. Pignatelli, A. Arons, S. Tonegawa, Engram Cells Retain Memory Under Retrograde Amnesia. Science. 348, 1007-1013 (2015).

8. A. Hayashi-Takagi, S. Yagishita, M. Nakamura, F. Shirai, Y. I. Wu, A. L. Loshbaugh, B. Kuhlman, K. M. Hahn, H. Kasai, Labelling and optical erasure of synaptic memory traces in the motor cortex. Nature. 525, 333-338 (2015).

9. A. Frick, J. Magee, D. Johnston, LTP is accompanied by an enhanced local excitability of pyramidal neuron dendrites. Nat Neurosci. 7, 126-135 (2004).

10. A. Govindarajan, I. Israely, S.-Y. Huang, S. Tonegawa, The dendritic branch is the preferred integrative unit for protein synthesis-dependent LTP. Neuron. 69, 132-146 (2011).

11. A. Govindarajan, R. J. Kelleher, S. Tonegawa, A clustered plasticity model of long-term memory engrams. Nat Rev Neurosci. 7, 575-583 (2006).

12. A. Losonczy, J. K. Makara, J. C. Magee, Compartmentalized dendritic plasticity and input feature storage in neurons. Nature. 452, 436-441 (2008).

13. C. D. Harvey, K. Svoboda, Locally dynamic synaptic learning rules in pyramidal neuron dendrites. Nature. 450, 1195-1200 (2007).

14. G. Kastellakis, A. J. Silva, P. Poirazi, Linking Memories across Time via Neuronal and Dendritic Overlaps in Model Neurons with Active Dendrites. Cell Reports. 17, 1491-1504 (2016). 
15. K. K. Cowansage, T. Shuman, B. C. Dillingham, A. Chang, P. Golshani, M. Mayford, Direct reactivation of a coherent neocortical memory of context. Neuron. 84, 432-441 (2014).

16. A. M. P. Miller, A. C. Serrichio, D. M. Smith, Dual-Factor Representation of the Environmental Context in the Retrosplenial Cortex. Cerebral Cortex. 31, 2720-2728 (2021).

17. G. Buzsáki, Neural syntax: cell assemblies, synapsembles and readers. Neuron. 68, 362385 (2010).

18. K. D. Harris, J. Csicsvari, H. Hirase, G. Dragoi, G. Buzsáki, Organization of cell assemblies in the hippocampus. Nature. 424, 552-556 (2003).

19. C. Dejean, J. Courtin, N. Karalis, F. Chaudun, H. Wurtz, T. C. M. Bienvenu, C. Herry, Prefrontal neuronal assemblies temporally control fear behaviour. Nature. 535, 420-424 (2016).

20. G. Girardeau, I. Inema, G. Buzsáki, Reactivations of emotional memory in the hippocampus-amygdala system during sleep. Nat Neurosci. 20, 1634-1642 (2017).

21. M. El-Gaby, H. M. Reeve, V. Lopes-dos-Santos, N. Campo-Urriza, P. V. Perestenko, A. Morley, L. A. M. Strickland, I. P. Lukács, O. Paulsen, D. Dupret, An emergent neural coactivity code for dynamic memory. Nat Neurosci. 24, 694-704 (2021).

22. Hebb, D. O. The organization of behavior: A neuropsychological theory. New York: John Wiley and Sons, Inc., 1949. 335 p. \$4.00. Science Education. 34, 336-337 (1950).

23. G. P. Gava, S. B. McHugh, L. Lefèvre, V. Lopes-dos-Santos, S. Trouche, M. El-Gaby, S. R. Schultz, D. Dupret, Integrating new memories into the hippocampal network activity space. Nat Neurosci. 24, 326-330 (2021).

24. H. Chang, I. M. Esteves, A. R. Neumann, J. Sun, M. H. Mohajerani, B. L. McNaughton, Coordinated activities of retrosplenial ensembles during resting-state encode spatial landmarks. Philosophical Transactions of the Royal Society B: Biological Sciences. 375, 20190228 (2020).

25. L. G. Reijmers, B. L. Perkins, N. Matsuo, M. Mayford, Localization of a stable neural correlate of associative memory. Science. 317, 1230-1233 (2007).

26. A. P. Yiu, V. Mercaldo, C. Yan, B. Richards, A. J. Rashid, H.-L. L. Hsiang, J. Pressey, V. Mahadevan, M. M. Tran, S. A. Kushner, M. A. Woodin, P. W. Frankland, S. A. Josselyn, Neurons Are Recruited to a Memory Trace Based on Relative Neuronal Excitability Immediately before Training. Neuron. 83, 722-735 (2014).

27. K. Abdou, M. Shehata, K. Choko, H. Nishizono, M. Matsuo, S. Muramatsu, K. Inokuchi, Synapse-specific representation of the identity of overlapping memory engrams. Science. 360, 1227-1231 (2018).

28. J. Voigts, M. T. Harnett, Somatic and Dendritic Encoding of Spatial Variables in Retrosplenial Cortex Differs during 2D Navigation. Neuron. 105, 237-245.e4 (2020). 
29. A. C. Frank, S. Huang, M. Zhou, A. Gdalyahu, G. Kastellakis, T. K. Silva, E. Lu, X. Wen, P. Poirazi, J. T. Trachtenberg, A. J. Silva, Hotspots of dendritic spine turnover facilitate clustered spine addition and learning and memory. Nat Commun. 9, 422 (2018).

30. C. Andreassi, A. Riccio, To localize or not to localize: mRNA fate is in 3'UTR ends. Trends in Cell Biology. 19, 465-474 (2009).

31. D. O. Wang, K. C. Martin, R. S. Zukin, Spatially restricting gene expression by local translation at synapses. Trends Neurosci. 33, 173-182 (2010).

32. S. Minoshima, B. Giordani, S. Berent, K. A. Frey, N. L. Foster, D. E. Kuhl, Metabolic reduction in the posterior cingulate cortex in very early Alzheimer's disease. Annals of Neurology. 42, 85-94 (1997).

33. G. Pengas, J. R. Hodges, P. Watson, P. J. Nestor, Focal posterior cingulate atrophy in incipient Alzheimer's disease. Neurobiology of Aging. 31, 25-33 (2010).

34. L. Petreanu, T. Mao, S. M. Sternson, K. Svoboda, The subcellular organization of neocortical excitatory connections. Nature. 457, 1142-1145 (2009).

35. M. P. Forrest, E. Parnell, P. Penzes, Dendritic structural plasticity and neuropsychiatric disease. Nat Rev Neurosci. 19, 215-234 (2018).

36. H. Kasai, N. E. Ziv, H. Okazaki, S. Yagishita, T. Toyoizumi, Spine dynamics in the brain, mental disorders and artificial neural networks. Nat Rev Neurosci. 22, 407-422 (2021).

37. S. Kim, N. Kim, J. Lee, S. Kim, J. Hong, S. Son, W. Do Heo, Dynamic Fas signaling network regulates neural stem cell proliferation and memory enhancement. Science Advances. 6, eaaz9691.

38. Miniscope-DAQ-Cypress-firmware (Aharoni Lab, 2021; https://github.com/AharoniLab/Miniscope-DAQ-Cypress-firmware).

39. E. A. Pnevmatikakis, A. Giovannucci, NoRMCorre: An online algorithm for piecewise rigid motion correction of calcium imaging data. Journal of Neuroscience Methods. 291, 83-94 (2017).

40. P. Zhou, S. L. Resendez, J. Rodriguez-Romaguera, J. C. Jimenez, S. Q. Neufeld, A. Giovannucci, J. Friedrich, E. A. Pnevmatikakis, G. D. Stuber, R. Hen, M. A. Kheirbek, B. L. Sabatini, R. E. Kass, L. Paninski, Efficient and accurate extraction of in vivo calcium signals from microendoscopic video data. eLife. 7, e28728 (2018).

41. G. Etter, MiniscopeAnalysis (2021; https://github.com/etterguillaume/MiniscopeAnalysis).

42. J. Friedrich, P. Zhou, L. Paninski, Fast online deconvolution of calcium imaging data. PLOS Computational Biology. 13, e1005423 (2017).

43. K. Z. Tanaka, H. He, A. Tomar, K. Niisato, A. J. Y. Huang, T. J. McHugh, The hippocampal engram maps experience but not place. Science. 361, 392-397 (2018). 
bioRxiv preprint doi: https://doi.org/10.1101/2021.10.28.466343; this version posted December 7, 2021. The copyright holder for this preprint

(which was not certified by peer review) is the author/funder, who has granted bioRxiv a license to display the preprint in perpetuity. It is made available under aCC-BY-ND 4.0 International license.

44. M. Pachitariu, C. Stringer, S. Schröder, M. Dipoppa, L. F. Rossi, M. Carandini, K. D. Harris, "Suite2p: beyond 10,000 neurons with standard two-photon microscopy" (2016), p. 061507. 